\title{
Prone Position Improves Lung Mechanical Behavior and Enhances Gas Exchange Efficiency in Mechanically Ventilated Chronic Obstructive Pulmonary Disease Patients
}

\author{
Spyros D. Mentzelopoulos, MD, DEAA, Spyros G. Zakynthinos, MD, PhD, \\ Charris Roussos, MD, PhD, Maria J. Tzoufi, MD, DEAA, \\ and Argyris S. Michalopoulos, MD, FCCM
}

Department of Intensive Care Medicine, Henry Dunant General Hospital; and Evangelismos General Hospital, Athens, Greece

Pronation might favorably affect respiratory system (rs) mechanics and function in volume-controlled, mode-ventilated chronic obstructive pulmonary disease (COPD) patients. We studied 10 COPD patients, initially positioned supine (baseline supine [supine $\left._{\mathrm{BAS}}\right]$ ) and then randomly and consecutively changed to protocol supine (supine ${ }_{\text {PROT }}$ ), semirecumbent, and prone positions. Rs mechanics and inspiratory work $\left(\mathrm{W}_{\mathrm{I}}\right)$ were assessed at baseline $(0.6 \mathrm{~L})$ (all postures) and sigh $(1.2 \mathrm{~L})$ (supine $_{\mathrm{BAs}}$ excluded) tidal volume (VT) with rapid airway occlusion during constant-flow inflation. Hemodynamics and gas exchange were assessed in all postures. There were no complications. Prone positioning resulted in (a) increased dynamic-static chest wall (cw) elastance (at both VTS) and improved oxygenation versus supine BAS, $_{\text {supine }}$ PROT, and semirecumbent, (b) decreased additional lung (L) resistance-elastance versus supine $_{\mathrm{PROT}}$ and semirecumbent at sigh $\mathrm{VT}_{\text {, }}$ (c) decreased L-static elastance (at both VTs) and improved $\mathrm{CO}_{2}$ elimination versus

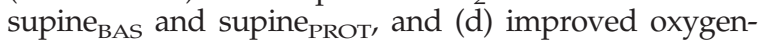
ation versus all other postures. Semirecumbent positioning increased mainly additional cw-resistance versus supine $_{\text {BAS }}$ and supine PROT $_{\text {at baseline. }}$ at $\mathrm{W}_{\mathrm{I}^{-}}$-subcomponent changes were consistent with changes in rs, $\mathrm{cW}$, and L mechanical properties. Total $\mathrm{rs}-\mathrm{W}_{\mathrm{I}}$ and hemodynamics were unaffected by posture change. After pronation, five patients were repositioned supine (su-

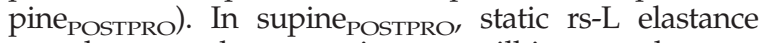
were lower, and oxygenation was still improved versus

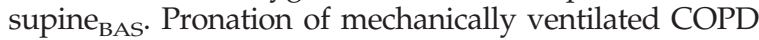
patients exhibits applicability and effectiveness and improves oxygenation and sigh-L mechanics versus semirecumbent ("gold standard") positioning.

(Anesth Analg 2003;96:1756-67)
D epending on respiratory system (rs) pathology, body posture differentially affects chest wall (cw), lung (L) mechanics, and gas exchange during artificial respiration $(1,2)$. In chronic obstructive pulmonary disease (COPD), posture change rs-effects are still unclear. Severe COPD is often associated with cor pulmonale, potentially causing L-compression and L-elastance increase, especially in the supine position. We theorized that in mechanically ventilated COPD patients, prone positioning may reduce L-elastance, increase cw-elastance, and improve gas exchange efficiency relative to supine and semirecumbent ("gold standard") positioning.

Accepted for publication February 10, 2003

Address correspondence and reprint requests to Spyros D. Mentzelopoulos, MD, DEAA, 12 Ioustinianou St., 11473 Athens, Greece. Address e-mail to sdm@hol.gr.

DOI: 10.1213/01.ANE.0000064282.79068.1E

\section{Methods}

IRB approval, nonwritten patient consent (whenever feasible), and informed written next-of-kin consent (always) were obtained $(1,3)$. We studied 10 moderate-to-severe COPD patients (4) (Table 1), orotracheally intubated (8.0-8.5 mm internal diameter [ID], 26-cm long endotracheal tube [ETT], Portex, UK) mechanically ventilated (Siemens 300C, Germany) because of acute respiratory failure (ARF) $\left(\mathrm{PaO}_{2}\right.$-inspired $\mathrm{O}_{2}$ fraction $\left[\mathrm{FIO}_{2}\right], 75-$ $184 \mathrm{~mm} \mathrm{Hg}$; $\mathrm{PaCO}_{2}, 61.0-101.4 \mathrm{~mm} \mathrm{Hg}$ ) secondary to severe, acute bronchitis (5).

Exclusion criteria were left ventricular failure, acute myocardial ischemia, L-lobar atelectasis, pneumonia, sepsis, pulmonary embolism, and mean pulmonary artery pressure (MPAP) $\geq 30 \mathrm{~mm} \mathrm{Hg}$. During the $4.5-$ to 5-h study period, patient care was provided by a physician uninvolved in the study. Any new or additional administration of IV fluid boluses, 
Table 1. Individual Patient Characteristics Before and After Hospital Admission and Initial Inhospital Management

\begin{tabular}{|c|c|c|c|c|c|c|c|c|}
\hline $\begin{array}{l}\text { Patent } \\
\text { no. }\end{array}$ & $\begin{array}{l}\text { Age } \\
(y r)\end{array}$ & Sex & $\begin{array}{c}\text { Body } \\
\text { mass } \\
\text { index } \\
\left(\mathrm{kg} / \mathrm{m}^{2}\right)\end{array}$ & $\begin{array}{l}\text { Smoking } \\
\text { (pack-yr) }^{\mathrm{a}}\end{array}$ & $\begin{array}{c}\text { Predominant } \\
\text { component of COPD }\end{array}$ & Comorbidity & $\begin{array}{c}\text { Evolution } \\
\text { of COPD } \\
(y r)\end{array}$ & $\begin{array}{c}\text { No. of } \\
\text { exacerbations } \\
\text { within } \\
\text { preceding } 12 \\
\text { mo }\end{array}$ \\
\hline 1 & 59 & Male & 24.2 & 46 & Pulmonary emphysema & HT & 9 & 1 \\
\hline 2 & 69 & Female & 22.4 & 102 & Chronic bronchitis & $\mathrm{HT}, \mathrm{AF}^{\mathrm{c}}, \mathrm{CVD}$ & 16 & 1 \\
\hline 3 & 58 & Male & 23.5 & 105 & Chronic bronchitis & None & 7 & 2 \\
\hline 4 & 71 & Male & 27.5 & 81 & Chronic bronchitis & $\mathrm{HT}, \mathrm{CAD}, \mathrm{MD}$ & 13 & 1 \\
\hline 5 & 67 & Male & 32.3 & 69 & Chronic bronchitis & $\mathrm{HT}, \mathrm{DM}, \mathrm{OB}, \mathrm{CAD}$ & 26 & 5 \\
\hline 6 & 74 & Female & 22.5 & 50 & Chronic bronchitis & $\mathrm{HT}, \mathrm{MAT}^{\mathrm{e}}$ PVD, $\mathrm{MD}^{\mathrm{d}}$ & 17 & 2 \\
\hline 7 & 56 & Male & 24.4 & 80 & Chronic bronchitis & None & 8 & 3 \\
\hline 8 & 61 & Male & 19.2 & 115 & Pulmonary emphysema & HT & 10 & 4 \\
\hline 9 & 63 & Female & 27.2 & 86 & Chronic bronchitis & HT & 14 & 2 \\
\hline 10 & 72 & Male & 27.1 & 80 & Chronic bronchitis & HT, DM, CAD, PVD, CVD & 23 & 2 \\
\hline
\end{tabular}

\begin{tabular}{|c|c|c|c|c|c|c|c|}
\hline $\begin{array}{l}\text { Patient } \\
\text { no. }\end{array}$ & 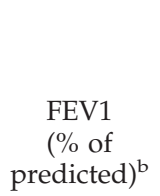 & $\begin{array}{c}\text { FVC } \\
(\% \text { of } \\
\text { predicted })^{\mathrm{b}}\end{array}$ & 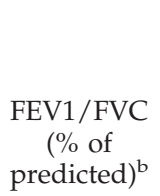 & $\begin{array}{l}\text { Admitted to the ICU from } \\
\text { hospital ward/emergency } \\
\text { room, before/after ETI }\end{array}$ & $\begin{array}{l}\text { APACHE II } \\
\text { score just } \\
\text { before ETI }\end{array}$ & $\begin{array}{l}\text { Initial ICU airway/ } \\
\text { ventilatory } \\
\text { management }\end{array}$ & $\begin{array}{l}\text { Probable etiology of } \\
\text { acute bronchitis; } \\
\text { microorganisms were } \\
\text { isolated in PSB and } \\
\text { TBAs or identified by } \\
\text { seroconversiong }\end{array}$ \\
\hline 1 & 31.0 & 58.7 & 52.8 & Hospital ward, before ETI & 18 & NIV (duration, $3 \mathrm{~h})^{\mathrm{f}}$ & Hemophilus influenzae \\
\hline 2 & 44.8 & 74.1 & 60.5 & Hospital ward, before ETI & 31 & ETI, VC ventilation & Hemophilus influenzae \\
\hline 3 & 23.8 & 50.3 & 47.3 & Emergency room, after ETI & 27 & VC ventilation & Hemophilus influenzae \\
\hline 4 & 28.1 & 46.8 & 60.0 & Emergency room, after ETI & 29 & VC ventilation & Pseudomonas aeruginosa \\
\hline 5 & 21.7 & 44.7 & 48.6 & Emergency room, after ETI & 34 & VC ventilation & $\begin{array}{c}\text { Hemophilus influenzae, } \\
\text { Enterobacter cloacae }\end{array}$ \\
\hline 6 & 42.3 & 81.6 & 51.8 & Emergency room, after ETI & 30 & VC ventilation & $\begin{array}{l}\text { Moraxella catarrhalis, } \\
\text { Pseudomonas } \\
\text { aeruginosa }\end{array}$ \\
\hline 7 & 26.4 & 48.0 & 55.0 & Hospital ward, before ETI & 27 & ETI, VC ventilation & $\begin{array}{l}\text { Moraxella catarrhalis, } \\
\text { Influenza virus }\end{array}$ \\
\hline 8 & 22.6 & 55.1 & 41.1 & Emergency room, after ETI & 27 & VC ventilation & $\begin{array}{l}\text { Streptococcus } \\
\text { pneumoniae, } \\
\text { Pseudomonas } \\
\text { aeruginosa }\end{array}$ \\
\hline 9 & 39.8 & 63.6 & 62.5 & Hospital ward, before ETI & 28 & NIV (duration, $2.5 \mathrm{~h}$ ) ${ }^{\mathrm{f}}$ & $\begin{array}{l}\text { Pseudomonas } \\
\text { aeruginosa, } \\
\text { Chlamydia } \\
\text { pneumoniae }\end{array}$ \\
\hline 10 & 29.8 & 44.4 & 67.0 & Hospital ward, before ETI & 28 & NIV (duration, $1 \mathrm{~h})^{\mathrm{f}}$ & $\begin{array}{l}\text { Streptococcus } \\
\text { pneumoniae }\end{array}$ \\
\hline
\end{tabular}

There were no patients with an already existing tracheostomy. The duration of mechanical ventilation until study protocol initiation ranged within $19.2-54.8 \mathrm{~h}$.

$\mathrm{COPD}=$ chronic obstructive pulmonary disease; $\mathrm{HT}=$ hypertension (primary); $\mathrm{AF}=$ atrial fibrillation; CVD = cerebrovascular disease; CAD = coronary artery disease; $\mathrm{MD}=$ major depression; $\mathrm{DM}=$ diabetes mellitus; $\mathrm{OB}=$ obesity; $\mathrm{MAT}=$ multifocal atrial tachycardia; $\mathrm{PVD}=$ peripheral vascular disease; FEV 1 and FVC = forced expiratory volume in $1 \mathrm{~s}$ and forced vital capacity, respectively (predicted values were those of Hankinson JL, Odencrantz JR, Fedan JB. Spirometric reference values from a sample of the general U.S. population. Am J Respir Crit Care Med 1999;159:178-87); ICU = intensive care unit; ETI = endotracheal intubation; APACHE = acute physiology and chronic health evaluation; NIV $=$ noninvasive ventilation; $\mathrm{VC}=$ volume-controlled; $\mathrm{PSB}=$ protected specimen brush (cutoff value for positive microorganism isolation, $10^{2}$ colony forming $\mathrm{U} / \mathrm{mL}$ ); TBAs = tracheobronchial aspirates (cutoff value for positive microorganism isolation, $10^{5}$ colony forming $\mathrm{U} / \mathrm{mL}$ ).

a All patients were current or ex smokers. ${ }^{b}$ Presented spirometric values were determined during the period of clinical stability that preceded the episode of acute bronchitis reported herein. ${ }^{\mathrm{c}}$ Asymptomatic on warfarin, digoxin, and verapamil. ${ }^{\mathrm{d}}$ Diagnosed with the Geriatric Mental State Schedule. ${ }^{\mathrm{e}}$ Previous history of stress-related, recurrent episodes, which were responsive to IV magnesium. ${ }^{\mathrm{f}}$ Ventilator settings were inspired $\mathrm{O}_{2}$ fraction of $0.30-0.60$; pressure support level

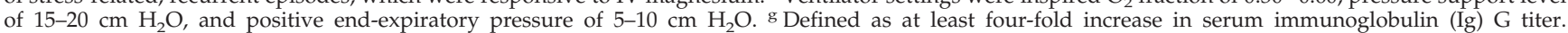
${ }^{h}$ Seroconversion as just defined was considered as diagnostic. ${ }^{i}$ Seroconversion as just defined reaching a titer of $\geq 1: 512$ or a serum IgM titer of $\geq 1: 32$ was considered as diagnostic.

inotropes, antipyretics, vasodilators, antiarrhythmic treatment, diuretics, insulin, or bronchodilators would cause patient exclusion. Electrocardiographic lead II, peripheral intraarterial, and pulmonary artery (Hands off ${ }^{\circledR}$ Infusion Port Thermodilution Catheter, Arrow, Reading, PA) pressures, urinary bladder temperature (Mon-a-therm ${ }^{\mathrm{TM}}$ Foley-Temp ${ }^{\mathrm{TM}}$, Mallinckrodt, St Louis, $\mathrm{MO}$ ), and peripheral $\mathrm{O}_{2}$ saturation $\left(\mathrm{SO}_{2}\right)$ were monitored continuously.
After replacement of any enteral nutrition by a parenteral of identical nutrient-composition and administration rate (with subsequent propofol infusion-related lipid intake taken into account), gastric contents' evacuation by suction, nasogastric tube removal, and hydroxyethyl starch administration $(3-5 \mathrm{~mL} / \mathrm{kg})$, patients were placed in a baseline supine (supine BAS $_{\text {) position }}$ (90 degree inclination). Anesthesia and neuromuscular blockade were induced and maintained throughout the 
study period with propofol-fentanyl and cisatracurium, respectively. Full train-of-four inhibition (facial nerve stimulation) was always accomplished. Baseline ventilator settings (volume control mode) were: tidal volume $(\mathrm{VT})$ of $0.6 \pm 0.02 \mathrm{~L}$; breaths $/ \mathrm{min}, 18.0 \pm 0.7$; inspiratory time-to-total respiratory cycle length ratio, $0.20 \pm 0.01$; inspiratory flow $(\mathrm{V} / \mathrm{s}), 0.91 \pm 0.02 \mathrm{~L} / \mathrm{s}$; plateau pressure time, $0 \mathrm{~s}$; positive end-expiratory pressure (PEEP), $0 \mathrm{~cm}$ $\mathrm{H}_{2} \mathrm{O}$; and $\mathrm{FIO}_{2}, 0.6$.

$\mathrm{V} / \mathrm{s}$ was measured with a heated pneumotachograph and a differential pressure transducer, and VT was obtained by V/s-signal integration (6). Tracheal pressure (Paw) was measured with a 1.5-mm ID, $50-\mathrm{cm}$ long catheter placed $2-3 \mathrm{~cm}$ past the ETT-tip and a pressure transducer (6). Esophageal pressure (Pes) and gastric pressure (Pga) were measured with a thin-walled latex double balloon-catheter system (6); proximal and distal balloons were placed in the midesophagus and stomach and inflated with 0.5 and $1.0 \mathrm{~mL}$ of air, respectively. Each balloon-catheter was proximally connected to a pressure transducer (6). Correct esophageal balloon placement was verified just before the cisatracurium administration by occlusion test (7). Paw-Pes difference yielded transpulmonary pressure (PL). After analog-to-digital conversion (sample rate, $200 \mathrm{~Hz}$ ), variable-data were stored on IBM-type computer hard disk for later-on-analysis with a dedicated program (2). Breathing circuit modifications were as previously described (6).

Respiratory mechanics were assessed with constant $\mathrm{V} / \mathrm{s}$ rapid airway occlusion (6) in the supine BAS, $_{\text {, protocol }}$

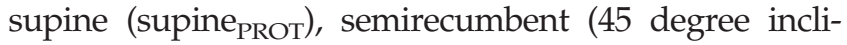
nation), and prone positions. The latter three postures' order was randomized a priori for all 10 patients with the Research Randomizer (http://www. randomizer.org/form.htm). Patients remained in each posture for 65-75 $\mathrm{min}$. Supine-to-prone and prone-tosupine turning were performed by six attendants with an ETT and pressure-measuring devices manually immobilized and temporarily disconnected from breathing circuit (for $\leq 20 \mathrm{~s}$ ) and pressure transducers, respectively. After patient turning, ETT displacement was excluded by capnography, breath soundauscultation, and unchanged insertion lengthconfirmation (used also for exclusion of pressuremeasuring devices displacement). After pronation, abdominal movement-restriction was minimized (2).

Apart from nonbaseline test breaths, described later, only previously described baseline ventilation

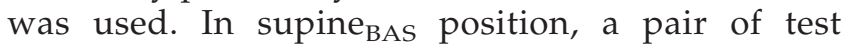
breaths (VT, $0.6 \mathrm{~L}$ [baseline]; square-wave V/s, 0.91 $\mathrm{L} / \mathrm{s}$ ) were administered within 45-55 min after neuromuscular blockade institution. Within 45-65 min after assumption of each studied posture, test breaths with constant, square-wave V/s $(0.91 \mathrm{~L} / \mathrm{s})$ and VT randomly varied from baseline to $0.2,0.4$,
$0.8,1.0$, and 1.2 (sigh) L were administered twice. Test breaths were separated by brief baseline ventilation periods (Fig. 1) (6).

For each rs component, variables were abbreviated as "defined variable abbreviation, rs component" (e.g., cw-intrinsic PEEP [PEEPi] or PEEPi,cw).

Baseline-ventilation PEEPi,rs was measured as the Paw-plateau during the test breath preceding $\sim 2$-s end-expiratory occlusion (EEO) referred to atmospheric pressure (Fig. 1). PEEPi, L, PEEPi,cw, and abdominal $\mathrm{cw}$-component (PEEPi,ab-cw) were measured as respective EEO-plateau pressures referred to their preocclusion values (8). PEEPi,ab-cw was always approximately zero. End-expiratory lung volume (EELV)-change ( $\triangle$ EELV) was measured as previously described (6).

EEO was followed by 4 to 5-s end-inspiratory occlusion (EIO), enabling determination of maximal pressure (Pmax), pressure immediately after EIOinitiation $\left(\mathrm{P}_{1}\right)$, and plateau pressure $\left(\mathrm{P}_{2}\right)$ during Paw-Pes computer-stored-data display, and of Pmax and $\mathrm{P}_{2}$ during Pga computer-stored-data display (Fig. 1). In accordance with PEEPi determinations, Paw values were referred to atmospheric pressure, whereas Pes-Pga values were referred to their pre-EEO values $(6,8)$. During EIO, Paw was unaffected by gas exchange (6).

1.2-L-VT test breaths were used as sigh breaths, which constitute effective recruitment maneuvers (3); other recruitment maneuvers were not used. According to randomized posture sequence, pronation was

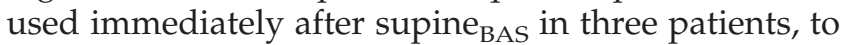
whom pre-prone sigh breaths were not administered. Sigh breaths were to be discontinued if $\mathrm{P}_{2}$ aw exceeded $45 \mathrm{~cm} \mathrm{H}_{2} \mathrm{O}$ (9). High $\mathrm{FiO}_{2}$ was selected because of possible participation of nonresponders to pronation (10) and to minimize hypoxemia-risk during $\triangle E E L V$ determinations and hemodynamic measurements. Any pronation-induced hypoxemia $\left(\mathrm{SO}_{2} \leq 90 \%\right)$ would result in protocol termination and body posture-ventilatory variable change.

In each posture, intravascular-pressure transducers (Abbott, Sligo, Ireland) were zeroed at right atrial level. Within 30-45 min after posture assumption, thermodilution cardiac output (CO), central venous pressure (CVP), and pulmonary artery wedge pressure (PAWP) were determined three times consecutively during respective 30 to 45 -s ETT disconnections from breathing circuit. ETT disconnections were initiated at end-inspiration and separated by two 5-min-lasting baseline ventilation intervals. Accordingly, heart rate and mean arterial blood pressure and MPAP values were averaged over each ETT-disconnection period. Just before each ETT disconnection, mixed venous and arterial 

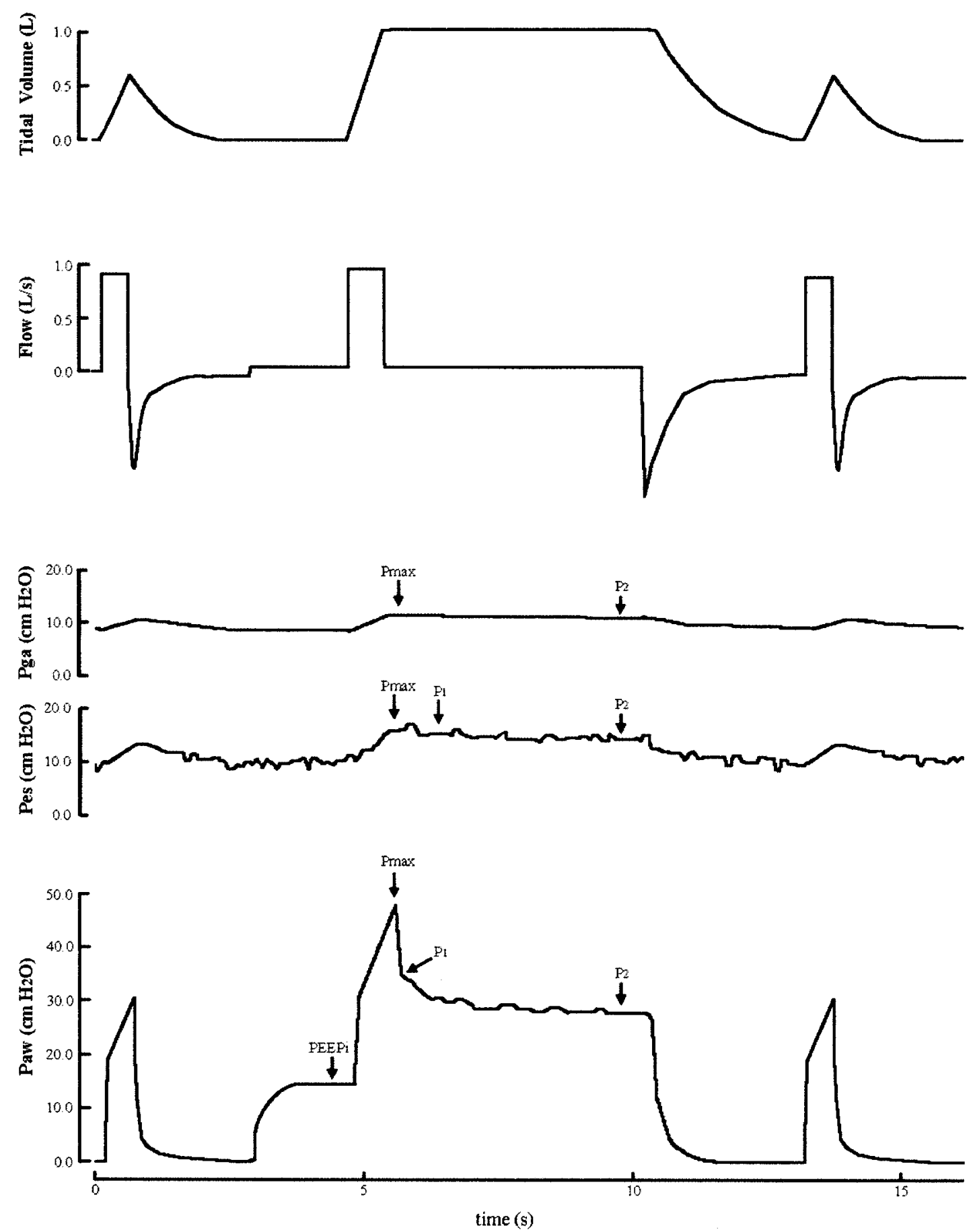

Figure 1. Tidal volume, inspiratory flow, and gastric (Pga), esophageal (Pes), and tracheal pressure (Paw) print-out of computer-stored-data display showing two baseline ventilation mechanical breaths (tidal volume $=0.6 \mathrm{~L}$ ) separated by a test breath (tidal volume $=1.0 \mathrm{~L}$ ). The presented variable-data originate from a representative study participant. The 1.88-s end-expiratory and the 4.60-s end-inspiratory airway occlusion (EIO) were performed before and just after the administration of the $1.0 \mathrm{~L}$ tidal volume, respectively. Note that on the displayed Pes tracing, characteristic perturbations caused by cardiac wall motion (i.e., cardiac oscillations) are present. Thus, Pmax was measured at the brief plateau preceding the first post-EIO cardiac oscillation, whereas $\mathrm{P}_{1}$ was measured at the brief plateau in-between the first and second post-EIO cardiac oscillation. The maximal amplitude of each EIO-oscillation was determined as the difference between oscillation's peak Pes-value and preceding plateau Pes-value. For each EIO-oscillation, Pes-increase rate was defined as Pes-change from preceding plateauto-peak value over time. In the presented test breath, Pes-increase rate was determinable in the 1st, 2nd, 3rd, 4th, and 6th post-EIO oscillations $(83 \%)$, because they were preceded by clearly identifiable plateaus. The same methodology was used in the analysis of all stored Pes-data. Pes-increase rate was determinable in $\geq 67 \%$ of EIO-oscillations of each analyzed test breath. PEEPi $=$ intrinsic positive end-expiratory pressure; $P$ max $=$ peak inspiratory pressure; $\mathrm{P}_{1}=$ pressure immediately after end-inspiratory airway occlusion; $\mathrm{P}_{2}=$ plateau inspiratory pressure. 

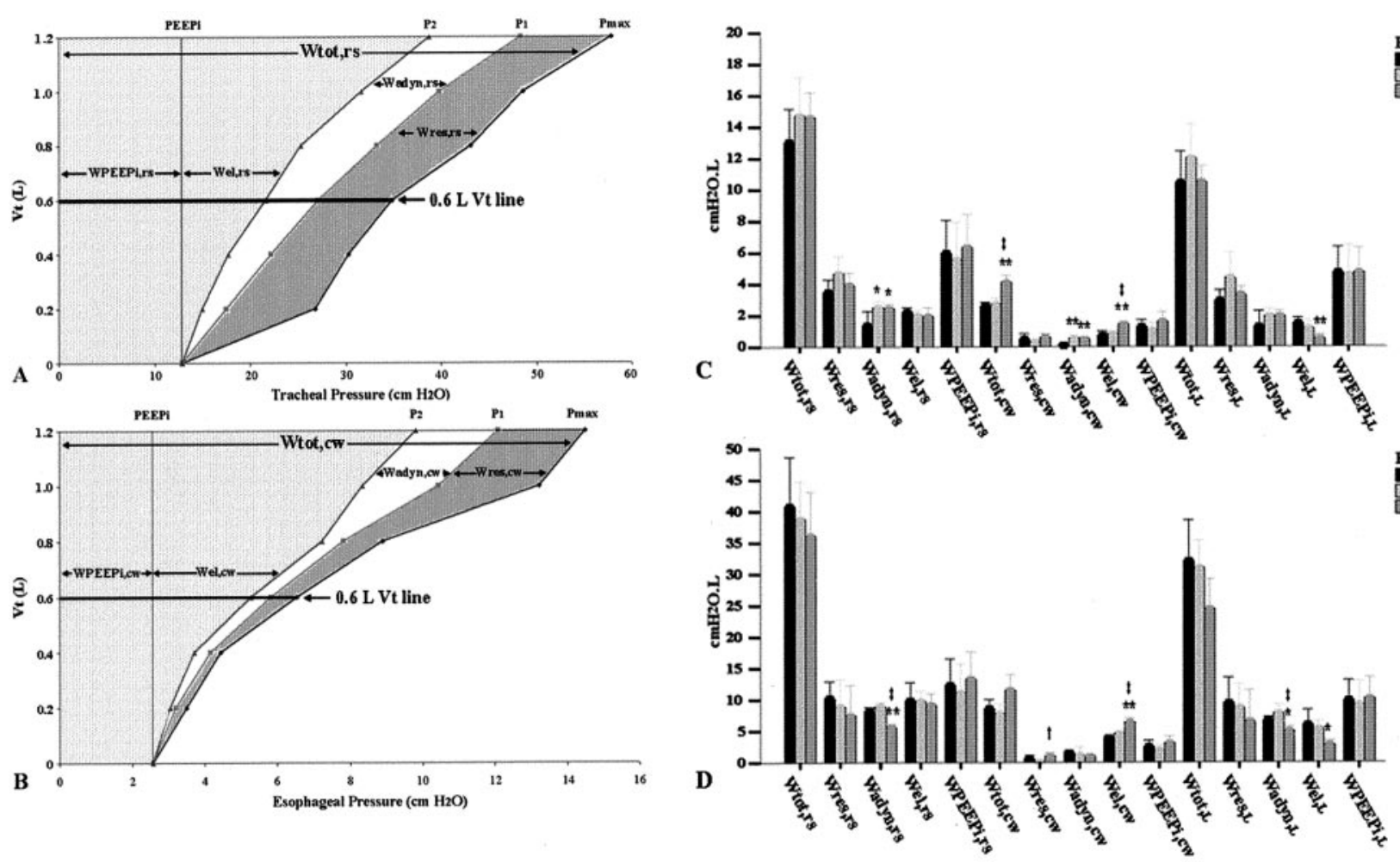

Figure 2. Methodology of determination of the static and dynamic components of the inspiratory work performed on the respiratory system (rs) (A) and chest wall (cw) (B) of a representative study participant; inspiratory work comparison-results at $0.6 \mathrm{~L}$ and $1.2 \mathrm{~L}$ inflation volume (VT) (C and D, respectively). (A and B) Work components corresponding to $0.6 \mathrm{~L}$ VT were determined by measuring the respective highlighted areas enclosed by the tracheal (A) and esophageal (B) pressure-volume curves labeled Pmax, $\mathrm{P}_{1}, \mathrm{P}_{2}$, and PEEPi, the volume and pressure axes, and the $0.6 \mathrm{~L}$ V lines. Work components corresponding to $1.2 \mathrm{~L}$ VT were determined by measuring the respective highlighted total areas subtended by the aforementioned pressure-volume curves. (C and D) Bars show mean values, and error bars show mean + sD. Comparison-results of inspiratory work subcomponents (measurement-unit, $\mathrm{cm} \mathrm{H}_{2} \mathrm{O} \cdot \mathrm{L}$ ) were thoroughly consistent in terms of presence of significant differences with the comparison-results of the rs, cw, and lung (L) mechanical properties they reflect (see also Appendix II and Results; Table 2; Figs. 3A,B,E,F). In the presented figure, inspiratory work subcomponents of each rs component are abbreviated as defined work subcomponent abbreviation, rs component (e.g., total work [Wtot] of the rs, Wtot,rs). Pmax $=$ peak inspiratory pressure; $\mathrm{P}_{1}=$ pressure immediately after end-inspiratory airway occlusion; $\mathrm{P}_{2}=$ plateau inspiratory pressure; PEEPi $=$ intrinsic positive end-expiratory pressure; Wtot = total work; Wres = resistive work, Wadyn = additional dynamic work; Wel = elastic work; WPEEPi $=$ work due to PEEPi. * $/ * *=$ significantly different versus protocol supine, $P<0.05 /<0.01$ (respectively); $\dagger / \ddagger=$ significantly different versus semirecumbent, $P<0.05 /<$ 0.01 (respectively).

blood gas (BG) samples were taken and analyzed immediately (ABL System 625; Radiometer, Copenhagen, Denmark). There were no appreciable differences among initial, second, and third BG values. Thus, BG analyses corresponded to baseline ventilation conditions. BG temperature corrections were not performed. Only BG analysis-derived $\mathrm{So}_{2}$ values were analyzed. Formula-derived variables included cardiac, systemic and pulmonary vascular resistance index, $\mathrm{O}_{2}$ consumption $\left(\mathrm{V}_{2}\right)$, respiratory quotient $(\mathrm{R})$, alveolar $\mathrm{PO}_{2}$, and shunt fraction $\left(\mathrm{Q}_{\mathrm{S}} / \mathrm{Q}_{\mathrm{T}}\right)$ (Appendix I). For each posture, only means of variable value-sets were analyzed.

$\mathrm{V} / \mathrm{s}, \mathrm{VT}, \mathrm{Paw}$, Pes, and Pga values of test breathpairs were stored and averaged in Microsoft Excel 2000. The following variable sets were determined at baseline and sigh Vт: (a) maximal (Rmax), ohmic (Rmin), and additional $(\Delta \mathrm{R})$ rs-component resistances (defined as corresponding Pmax- $\mathrm{P}_{2}$, Pmax-
$\mathrm{P}_{1}$, and $\mathrm{P}_{1}-\mathrm{P}_{2}$ differences divided by preceding $\mathrm{V} / \mathrm{s}$, respectively) and (b) dynamic (Edyn), static (Estat), and additional $(\Delta \mathrm{E})$ rs-component elastances (defined as corresponding $\mathrm{P}_{1}-\mathrm{PEEPi}, \mathrm{P}_{2}-\mathrm{PEEPi}$, and $\mathrm{P}_{1}-\mathrm{P}_{2}$ differences divided by preceding $\mathrm{VT}$, respectively). For the three protocol postures, pressurevolume curves were constructed, and total (Wtot), resistive, additional dynamic, elastic, and PEEPi inspiratory work per breath performed on each rs component were determined by respective surface area measurement in Autocad 2000 (Autodesk, San Rafael, CA) (Figs. 2A and B).

Variable-comparisons among body postures were conducted with repeated-measures analysis of variance, followed by Scheffé test for post hoc comparisons whenever appropriate. Significance was set at $P<$ 0.05 . Values are presented as mean \pm SD or grand mean* \pm SD. Statistical analysis was performed with SPSS 10.0 (SPSS Inc., Chicago, IL). 
Table 2. Respiratory System, Chest Wall, and Lung Mechanics During Baseline Ventilation (inflation volume $=0.6$ L) and Sigh-Equivalent (inflation volume $=1.2 \mathrm{~L}$ ) Test Breaths and Corresponding Ventilatory Variables

Baseline breath: VT $(\mathrm{L}), 0.6 \pm 0.01 ; \mathrm{f} / \mathrm{min}, 18.0 \pm 0.4 ; \mathrm{Ti}(\mathrm{s}), 0.66 \pm 0.02 ; \mathrm{V} / \mathrm{s}(\mathrm{L} / \mathrm{s}), 0.91 \pm 0.01 ;$ EEO time (s), $-2 ;$ EIO time (s), 4-5; PEEPe $\left(\mathrm{cm} \mathrm{H}_{2} \mathrm{O}\right), 0$

\begin{tabular}{|c|c|c|c|c|c|c|c|}
\hline Position, rs component & $\begin{array}{c}\mathrm{Rmax} \\
\left(\mathrm{cm} \mathrm{H} \mathrm{H}_{2} \mathrm{O} / \mathrm{L} / \mathrm{s}\right)\end{array}$ & $\begin{array}{c}\mathrm{Rmin} \\
\left(\mathrm{cm} \mathrm{H} \mathrm{H}_{2} \mathrm{O} / \mathrm{L} / \mathrm{s}\right)\end{array}$ & $\begin{array}{c}\Delta \mathrm{R} \\
\left(\mathrm{cmH}_{2} \mathrm{O} / \mathrm{L} / \mathrm{s}\right)\end{array}$ & $\begin{array}{c}\text { Edyn } \\
\left(\mathrm{cm} \mathrm{H} \mathrm{H}_{2} \mathrm{O} / \mathrm{L}\right)\end{array}$ & $\begin{array}{c}\text { Estat } \\
\left(\mathrm{cm} \mathrm{H} \mathrm{H}_{2} \mathrm{O} / \mathrm{L}\right)\end{array}$ & $\begin{array}{c}\Delta \mathrm{E} \\
\left(\mathrm{cm} \mathrm{H} \mathrm{H}_{2} \mathrm{O} / \mathrm{L}\right)\end{array}$ & $\begin{array}{c}\text { PEEPi } \\
\left(\mathrm{cm} \mathrm{H}_{2} \mathrm{O}\right)\end{array}$ \\
\hline Baseline supine, rs & $14.3 \pm 3.0$ & $9.1 \pm 2.7$ & $5.2 \pm 1.0$ & $23.8 \pm 1.9$ & $16.0 \pm 1.9$ & $7.9 \pm 1.6$ & $9.5 \pm 3.1$ \\
\hline Baseline supine, $\mathrm{cw}$ & $1.4 \pm 0.7$ & $0.8 \pm 0.3$ & $0.6 \pm 0.4$ & $6.0 \pm 1.0$ & $5.2 \pm 1.0$ & $0.9 \pm 0.6$ & $2.1 \pm 0.8$ \\
\hline Baseline supine, L & $12.9 \pm 2.4$ & $8.3 \pm 2.5$ & $4.6 \pm 1.1$ & $17.8 \pm 1.9$ & $10.8 \pm 1.7$ & $7.0 \pm 1.6$ & $7.4 \pm 2.4$ \\
\hline Protocol supine, rs & $14.1 \pm 2.5$ & $9.2 \pm 2.6$ & $4.9 \pm 1.0$ & $22.1 \pm 2.8$ & $14.7 \pm 3.1$ & $7.4 \pm 1.5$ & $9.9 \pm 3.5$ \\
\hline Protocol supine, $\mathrm{cw}$ & $1.5 \pm 0.6$ & $0.9 \pm 0.3$ & $0.6 \pm 0.4$ & $6.1 \pm 0.9$ & $5.2 \pm 0.9$ & $0.9 \pm 0.6$ & $2.1 \pm 0.8$ \\
\hline Protocol supine, L & $12.6 \pm 2.1$ & $8.3 \pm 2.4$ & $4.3 \pm 1.0$ & $16.0 \pm 3.4$ & $9.5 \pm 3.2$ & $6.5 \pm 1.6$ & $7.8 \pm 2.9$ \\
\hline Semirecumbent, rs & $19.2 \pm 2.8 \| \dagger$ & $11.5 \pm 2.4$ & $7.7 \pm 1.3 \| \dagger$ & $26.2 \pm 3.2$ & $14.5 \pm 3.1$ & $11.7 \pm 2.0 \| \dagger$ & $9.3 \pm 3.9$ \\
\hline Semirecumbent, cw & $2.5 \pm 0.6$ & $0.8 \pm 0.3$ & $1.6 \pm 0.6 \| \dagger$ & $8.2 \pm 0.7 \| \dagger$ & $5.8 \pm 0.6$ & $2.5 \pm 1.0 \| \dagger$ & $1.7 \pm 0.7$ \\
\hline Semirecumbent, L & $16.7 \pm 2.4$ & $10.7 \pm 2.5$ & $6.1 \pm 1.1$ & $17.9 \pm 3.2$ & $8.7 \pm 2.7$ & $9.2 \pm 1.6$ & $7.6 \pm 3.2$ \\
\hline Prone, total rs & $17.2 \pm 3.5$ & $9.7 \pm 3.8$ & $7.5 \pm 0.5 \| \dagger$ & $25.8 \pm 2.7$ & $14.4 \pm 2.2$ & $11.4 \pm 0.8 \| \dagger$ & $10.5 \pm 3.9$ \\
\hline Prone, cw & $2.6 \pm 1.0$ & $1.1 \pm 0.5$ & $1.5 \pm 0.5 \| \dagger$ & $10.7 \pm 0.7 \|+\ddagger$ & $8.4 \pm 0.9 \| \dagger \neq$ & $2.3 \pm 0.8 \| \dagger$ & $2.7 \pm 1.0$ \\
\hline Prone, L & $14.2 \pm 2.9$ & $8.6 \pm 3.5$ & $6.0 \pm 1.0$ & $15.1 \pm 2.4$ & $6.0 \pm 1.4 \S^{*}$ & $9.1 \pm 1.4$ & $7.9 \pm 2.4$ \\
\hline
\end{tabular}

Sigh breath: Vт (L), $1.2 \pm 0.02 ; \mathrm{f} / \mathrm{min}, 9.0 \pm 0.2 ; \mathrm{Ti}(\mathrm{s}), 1.33 \pm 0.03 ; \mathrm{V} / \mathrm{s}(\mathrm{L} / \mathrm{s}), 0.91 \pm 0.02 ;$ EEO time (s), $-2 ;$ EIO time (s), 4-5; PEEPe $\left(\mathrm{cm} \mathrm{H}_{2} \mathrm{O}\right), 0$

\begin{tabular}{|c|c|c|c|c|c|c|c|}
\hline Position, rs component & 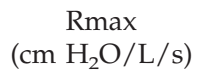 & $\begin{array}{c}\mathrm{Rmin} \\
\left(\mathrm{cm} \mathrm{H} \mathrm{H}_{2} \mathrm{O} / \mathrm{L} / \mathrm{s}\right)\end{array}$ & $\begin{array}{c}\Delta \mathrm{R} \\
\left(\mathrm{cmH}_{2} \mathrm{O} / \mathrm{L} / \mathrm{s}\right)\end{array}$ & $\begin{array}{c}\text { Edyn } \\
\left(\mathrm{cm} \mathrm{H} \mathrm{H}_{2} \mathrm{O} / \mathrm{L}\right)\end{array}$ & $\begin{array}{c}\text { Estat } \\
\left(\mathrm{cm} \mathrm{H} \mathrm{H}_{2} \mathrm{O} / \mathrm{L}\right)\end{array}$ & $\begin{array}{c}\Delta \mathrm{E} \\
\left(\mathrm{cm} \mathrm{H} \mathrm{H}_{2} \mathrm{O} / \mathrm{L}\right)\end{array}$ & $\begin{array}{c}\text { PEEPi } \\
\left(\mathrm{cm} \mathrm{H} \mathrm{H}_{2} \mathrm{O}\right)\end{array}$ \\
\hline Protocol supine, rs & $18.6 \pm 2.5$ & $10.5 \pm 1.1$ & $8.1 \pm 1.6$ & $27.5 \pm 3.0$ & $21.4 \pm 2.6$ & $6.1 \pm 1.2$ & $10.3 \pm 3.5$ \\
\hline Protocol supine, $\mathrm{cw}$ & $3.8 \pm 1.4$ & $0.8 \pm 0.3$ & $3.1 \pm 1.0$ & $7.6 \pm 1.1$ & $5.3 \pm 0.5$ & $2.3 \pm 0.8$ & $2.1 \pm 0.8$ \\
\hline Protocol supine, L & $14.7 \pm 2.5$ & $9.8 \pm 1.3$ & $5.0 \pm 1.5$ & $19.8 \pm 2.0$ & $16.1 \pm 2.3$ & $3.8 \pm 1.1$ & $8.2 \pm 2.7$ \\
\hline Semirecumbent, rs & $18.4 \pm 2.7$ & $9.3 \pm 0.2$ & $9.1 \pm 2.6$ & $26.4 \pm 4.6$ & $19.6 \pm 3.2$ & $6.9 \pm 2.0$ & $9.4 \pm 3.9$ \\
\hline Semirecumbent, cw & $2.6 \pm 0.7$ & $0.5 \pm 0.2$ & $2.1 \pm 0.7$ & $8.1 \pm 1.4$ & $6.6 \pm 1.3$ & $1.6 \pm 0.6$ & $1.7 \pm 0.7$ \\
\hline Semirecumbent, L & $15.8 \pm 2.7$ & $8.8 \pm 0.2$ & $7.0 \pm 2.7$ & $18.3 \pm 3.3$ & $13.0 \pm 2.8$ & $5.3 \pm 2.0$ & $7.7 \pm 3.2$ \\
\hline Prone, total rs & $12.1 \pm 2.3+\ddagger$ & $9.1 \pm 2.0$ & $3.0 \pm 1.3+\ddagger$ & $23.8 \pm 1.7$ & $21.6 \pm 2.2$ & $2.3 \pm 1.0+\ddagger$ & $11.1 \pm 3.6$ \\
\hline Prone, cw & $3.2 \pm 2.0$ & $1.5 \pm 0.6 \neq$ & $1.7 \pm 1.5$ & $11.7 \pm 1.3+\ddagger$ & $10.4 \pm 0.8+\ddagger$ & $1.3 \pm 1.2$ & $2.6 \pm 0.9$ \\
\hline Prone, L & $8.9 \pm 1.3$ & $7.6 \pm 1.7$ & $1.3 \pm 1.1^{*} \ddagger$ & $12.2 \pm 1.3+\ddagger$ & $11.1 \pm 1.4^{*}$ & $1.0 \pm 0.9^{*} \ddagger$ & $8.5 \pm 2.7$ \\
\hline
\end{tabular}

Values are mean $\pm \mathrm{SD}$.

$\mathrm{VT}$, inflation volume; f/min, ventilatory rate; Ti, inspiratory time; V/s, inspiratory flow; EEO, end-expiratory occlusion; EIO, end-inspiratory occlusion; PEEPe, externally applied positive end-expiratory pressure; rs, respiratory system; cw, chest wall; L, lung; Rmax, maximal (total) resistance; Rmin, ohmic resistance; $\Delta \mathrm{R}$, additional resiatance; Edyn, dynamic elastance; Estat, static elastance; $\Delta \mathrm{E}$, additional elastance; $\mathrm{PEEPi}$, intrinsic positive end-expiratory pressure.

${ }^{*} P<0.05$ significantly different versus protocol supine; $\dagger P<0.01$ significantly different versus protocol supine; $¥ P<0.01$ significantly different versus semirecumbent; $\S P<0.05$ significantly different versus baseline supine; $\| P<0.01$ significantly different versus baseline supine.

\section{Results}

Full data were obtained from all patients, and no protocol-related complications (e.g., pneumothoraxatelectasis, extubation-catheter removal, or patient injury) (10) occurred. Sigh breath $\mathrm{P}_{2}$ aw never exceeded $41.0 \mathrm{~cm} \mathrm{H}_{2} \mathrm{O}$. Nine patients were weaned from mechanical ventilation at $4.9 \pm 1.0$ days and discharged from the hospital $5.7 \pm 1.2$ days thereafter; one died of sepsis.

At baseline $\mathrm{VT}$, prone positioning resulted in (a) higher Edyn,cw and Estat,cw versus all other postures (all $P<0.01$ ), and (b) higher $\Delta \mathrm{R}, \mathrm{rs}, \Delta \mathrm{E}, \mathrm{rs}, \Delta \mathrm{R}, \mathrm{cw}$, and $\Delta \mathrm{E}, \mathrm{cW}$ and lower Estat,L versus supine $\mathrm{BAS}_{\mathrm{B}}$ and supine $_{\text {PROT }}(P<0.05-0.01)$; semirecumbent positioning resulted in higher Rmax,rs, $\Delta \mathrm{R}, \mathrm{rs}, \Delta \mathrm{E}, \mathrm{rs} \quad \Delta \mathrm{R}, \mathrm{cw}$, Edyn,cw, and $\Delta \mathrm{E}, \mathrm{cW}$ versus supine $\mathrm{BAS}_{\mathrm{B}}$ and supine $\mathrm{PROT}_{\mathrm{P}}$ (all $P<0.01$ ) (Table 2; Figs. 3A,B,E,F). At sigh VT, prone positioning resulted in (a) lower Rmax,rs, $\Delta \mathrm{R}, \mathrm{rs}$, $\Delta \mathrm{E}, \mathrm{rs}, \Delta \mathrm{R}, \mathrm{L}$, and $\Delta \mathrm{E}, \mathrm{L}$ and higher Edyn,cw and Estat, $\mathrm{CW}$ versus supine ${ }_{\mathrm{PROT}}$ and semirecumbent $(P<$ 0.05-0.01), (b) higher Rmin,cw versus semirecumbent $(P<0.01)$, and (c) lower Estat, $\mathrm{L}$ versus supine ${ }_{\text {PROT }}(P$ $<0.05)$. Inspiratory work comparison-results were consistent with our results on elastance, resistance, and PEEPi (Appendix II; Figs. 2C and D). Wtot,rs, which reflected total rs-impedance to mechanical breathing while VT increased from $0.2 \mathrm{~L}$ to sigh (Appendix II; Fig. 2A), was unaffected by posture change (Figs. 2C and D).

No significant changes were observed in $\triangle E E L V\left(0.41^{*}\right.$ $\pm 0.08 \mathrm{~L}), \Delta \mathrm{Pmax}$,ga at baseline and sigh $\mathrm{VT}\left(3.8^{*} \pm 1.0\right.$ and $6.3^{*} \pm 3.6 \mathrm{~cm} \mathrm{H}_{2} \mathrm{O}$, respectively), and $\Delta \mathrm{P}_{2}$,ga at baseline and sigh Vт $\left(3.4^{*} \pm 0.8\right.$ and $4.9^{*} \pm 3.8 \mathrm{~cm} \mathrm{H}_{2} \mathrm{O}$, respectively). Thus, body posture did not affect dynamic hyperinflation (6), Rmax,ab-cw, and Estat,ab-cw. Pga at rs relaxation volume (Vr) (11.1* $\pm 4.9 \mathrm{~cm} \mathrm{H}_{2} \mathrm{O}$ ) was also unaffected by posture change, suggesting lack of prone position-related abdominal compression.

Pes at EELV (pre-EEO value) and Vr were lower in prone versus supine BAS $_{\text {and }}$ andine PROT $_{\text {PROsitions }}$ $(10.7 \pm 2.1$ and $6.1 \pm 1.5$ versus $17.8 \pm 3.5$ and $16.9 \pm$ 3.0 and $13.3 \pm 4.0$ and $10.6 \pm 1.8 \mathrm{~cm} \mathrm{H}_{2} \mathrm{O}$, respectively; all $P<0.05$ ), indicating lack of comparability of absolute Pes-measurements. Pes at EELV exceeded Pes at 


\section{$\underline{\text { Tidal Volume }=0.6 \mathrm{~L}}$}

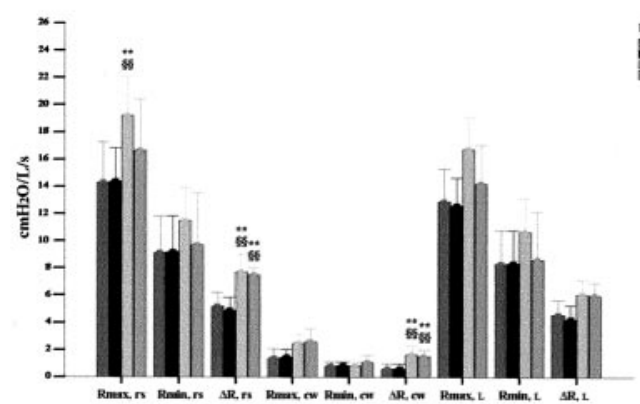

$\mathbf{A}$

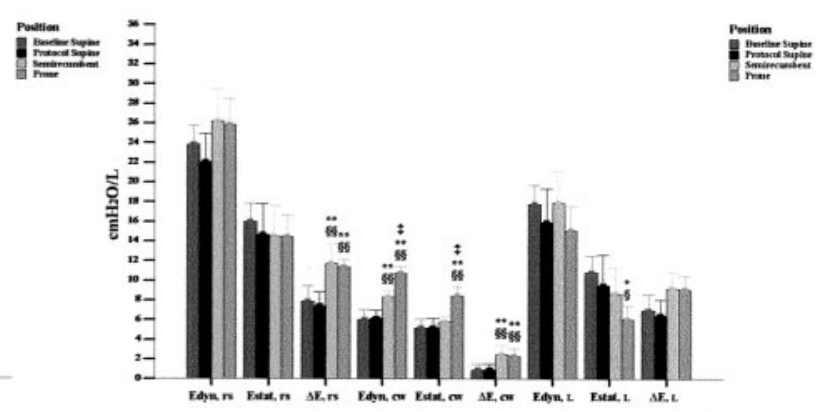

B

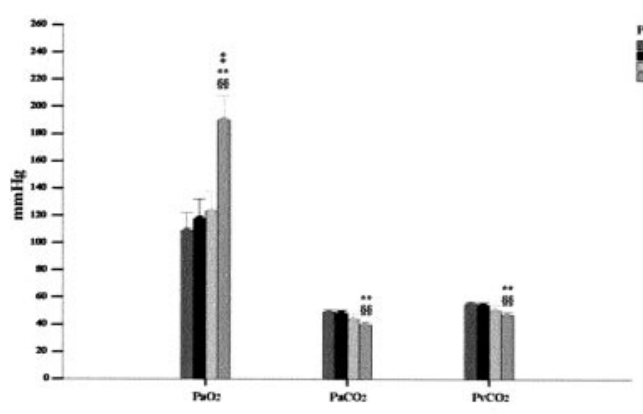

C
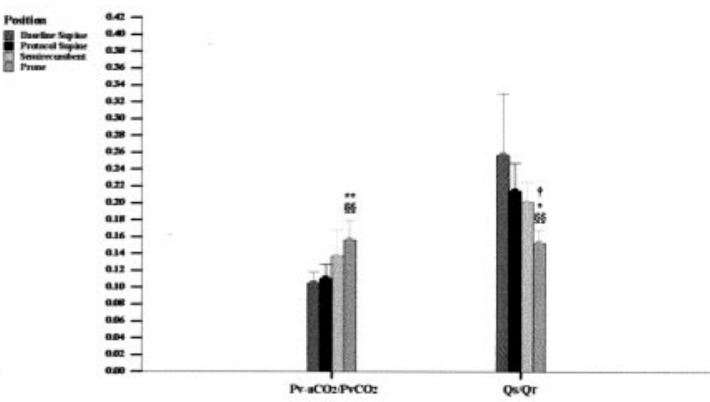

D

Tidal Volume $=1.2 \mathrm{~L}$

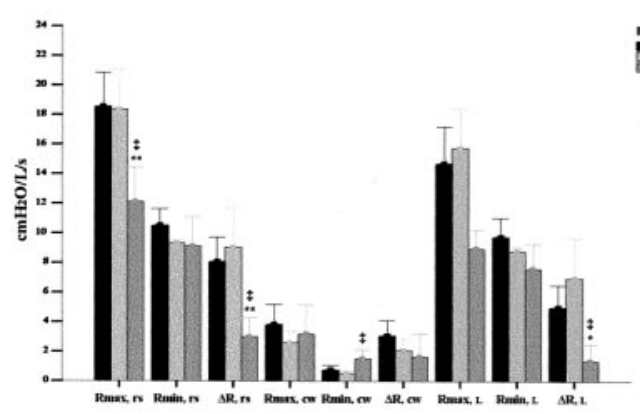

$\mathbf{E}$

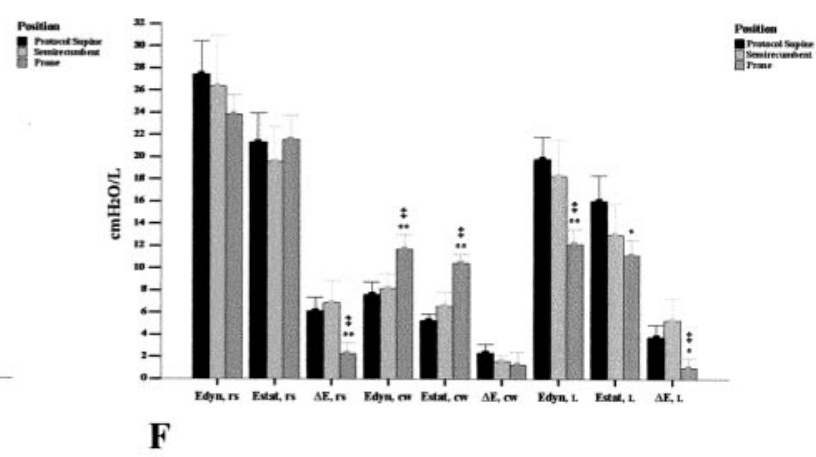

Figure 3. Results on resistance, elastance, and gas exchange obtained from the whole study group. Bars show mean values, and error bars show mean + SD; precise numerical values are given in Tables 2 and 3 . The presentation includes only variables that exhibited at least one posture change-related significant change (Tables 2 and 3). (A-D) Results on resistance, elastance, blood gases, and $\mathrm{CO}_{2}$ elimination and shunt fraction (respectively) obtained at baseline $(0.6 \mathrm{~L})$ tidal volume; (E and F) results on resistance and elastance (respectively) obtained at sigh (1.2 L) tidal volume. $\mathrm{rs}=$ respiratory system; $\mathrm{cw}=$ chest wall; $\mathrm{L}=$ lung; Rmax = maximal (total) resistance; Rmin = ohmic resistance; $\Delta \mathrm{R}=$ additional resistance because of tissue stress relaxation tension and/or L-time constant inequalities; Edyn = dynamic elastance; Estat $=$ static elastance; $\Delta \mathrm{E}=$ additional elastance due to tissue stress relaxation tension and/or L-time constant inequalities; Pa $=$ arterial gas partial pressure; $\mathrm{PV}=$ mixed venous gas partial pressure; $\mathrm{PV}-\mathrm{a}=$ mixed venous-to-arterial gas partial pressure difference; $\mathrm{Q}_{\mathrm{S}} / \mathrm{Q}_{\mathrm{T}}=$ shunt fraction. In the presented figure, elastance and resistance variables of each rs-component are abbreviated as already defined variable abbreviation, rs component (e.g., maximal [total] resistance [Rmax] of the rs, Rmax,rs). $\S / \S \S=$ significantly different versus baseline supine, $P<0.05 /<0.01$ (respectively); ${ }^{*}{ }^{* *}=$ significantly different versus protocol supine, $P<0.05 /<0.01$ (respectively); $† / \ddagger=$ significantly different versus semirecumbent, $P<0.05 /<0.01$ (respectively). 
Table 3. Hemodynamics and gas exchange. Gas exchange results correspond to baseline ventilation conditions (see also Methods)

\begin{tabular}{|c|c|c|c|c|c|c|c|c|c|c|c|c|c|}
\hline \multicolumn{14}{|c|}{ Hemodynamic variables } \\
\hline Body posture & $\begin{array}{c}\mathrm{HR} \\
\text { (beats/min) }\end{array}$ & $\begin{array}{c}\text { MAP } \\
(\mathrm{mm} \mathrm{Hg})\end{array}$ & $\begin{array}{c}\text { CVP } \\
(\mathrm{mm} \mathrm{Hg})\end{array}$ & $\begin{array}{l}\text { MPAP } \\
(\mathrm{mm} \mathrm{Hg})\end{array}$ & \multicolumn{2}{|c|}{$\begin{array}{l}\text { PAWP } \\
(\mathrm{mm} \mathrm{Hg})\end{array}$} & $\begin{array}{c}\mathrm{CI} \\
\left(\mathrm{L} \cdot \mathrm{min} \cdot \mathrm{m}^{2}\right)\end{array}$ & $\begin{array}{c}\mathrm{SvO}_{2} \\
(\%)\end{array}$ & \multicolumn{2}{|c|}{$\begin{array}{c}\mathrm{VO}_{2} \\
\left(\mathrm{~mL} \cdot \min \cdot \mathrm{m}^{2}\right)\end{array}$} & \multicolumn{2}{|c|}{$\begin{array}{c}\text { SVRI } \\
\left(\text { dynes's } \mathrm{s}^{\circ} \mathrm{cm}^{5} \cdot \mathrm{m}^{2}\right)\end{array}$} & $\begin{array}{c}\text { PVRI } \\
\left(\text { dynes' }^{\prime} \cdot \mathrm{cm}^{5} \cdot \mathrm{m}^{2}\right)\end{array}$ \\
\hline Baseline supine & $86 \pm 9$ & $81 \pm 11$ & $9 \pm 2$ & $24 \pm 3$ & \multicolumn{2}{|c|}{$13 \pm 2$} & $4.1 \pm 0.4$ & $82 \pm 2$ & \multicolumn{2}{|c|}{$140 \pm 19$} & \multicolumn{2}{|c|}{$1412 \pm 286$} & 209 \\
\hline Protocol supine & $86 \pm 9$ & $81 \pm 10$ & $9 \pm 2$ & $24 \pm 3$ & \multicolumn{2}{|c|}{$14 \pm 2$} & $4.2 \pm 0.5$ & $82 \pm 2$ & \multicolumn{2}{|c|}{$142 \pm 19$} & \multicolumn{2}{|c|}{$1388 \pm 268$} & 47 \\
\hline Semirecumbent & $93 \pm 9$ & $78 \pm 10$ & $9 \pm 2$ & $23 \pm 3$ & \multicolumn{2}{|c|}{$12 \pm 2$} & $4.1 \pm 0.5$ & $82 \pm 2$ & & \pm 20 & \multicolumn{2}{|c|}{$1367 \pm 288$} & $206 \pm 51$ \\
\hline Prone & $89 \pm 7$ & $80 \pm 9$ & $9 \pm 2$ & $25 \pm 4$ & \multicolumn{2}{|c|}{$13 \pm 2$} & $4.2 \pm 0.5$ & $82 \pm 2$ & & \pm 21 & \multicolumn{2}{|c|}{$1369 \pm 286$} & $216 \pm 59$ \\
\hline \multicolumn{14}{|c|}{ Gas exchange data } \\
\hline Body posture & $\begin{array}{c}\mathrm{PaO}_{2} \\
(\mathrm{~mm} \mathrm{Hg})\end{array}$ & \multicolumn{2}{|r|}{$\begin{array}{c}\mathrm{PaCO}_{2} \\
(\mathrm{~mm} \mathrm{Hg})\end{array}$} & \multicolumn{2}{|l|}{$\mathrm{pHa}$} & \multicolumn{2}{|c|}{$\begin{array}{c}\mathrm{PvO}_{2} \\
(\mathrm{~mm} \mathrm{Hg})\end{array}$} & \multicolumn{2}{|c|}{$\begin{array}{c}\mathrm{PvCO}_{2} \\
(\mathrm{~mm} \mathrm{Hg})\end{array}$} & \multicolumn{2}{|c|}{$\mathrm{pHv}$} & $\mathrm{Q}_{\mathrm{S}} / \mathrm{Q}_{\mathrm{T}}$ & $\begin{array}{l}\mathrm{PV}-\mathrm{aCO}_{2} / \\
\mathrm{PvCO}_{2}\end{array}$ \\
\hline Baseline st & $109 \pm 13$ & \multicolumn{2}{|c|}{$49.5 \pm 1.5$} & \multicolumn{2}{|c|}{$7.41 \pm 0.06$} & \multicolumn{2}{|c|}{$49.8 \pm 3.4$} & $55.2 \pm 1$ & & \multicolumn{2}{|c|}{$7.38 \pm 0.05$} & $0.26 \pm 0.07$ & $0.10 \pm 0.01$ \\
\hline Protocol supine & $118 \pm 14$ & \multicolumn{2}{|c|}{$48.5 \pm 2.0$} & \multicolumn{2}{|c|}{$7.42 \pm 0.07$} & \multicolumn{2}{|c|}{$51.5 \pm 4.3$} & $54.4 \pm 1$ & & \multicolumn{2}{|c|}{$7.38 \pm 0.05$} & $0.22 \pm 0.03$ & $0.11 \pm 0.02$ \\
\hline Semirecumbent & $123 \pm 15$ & \multicolumn{2}{|c|}{$43.5 \pm 4.5$} & \multicolumn{2}{|c|}{$7.44 \pm 0.06$} & \multicolumn{2}{|c|}{$51.4 \pm 4.2$} & $50.3 \pm 3$ & & $7.39 \pm$ & \pm 0.04 & $0.20 \pm 0.02$ & $0.14 \pm 0.03$ \\
\hline Prone & $190 \pm 18 \| \dagger$ & & $.0 \pm 2.0 \| \dagger$ & $7.45 \pm 0$ & .05 & 52.0 & \pm 3.5 & $47.4 \pm 2$ & $.0+\|$ & $7.39 \pm$ & \pm 0.04 & $0.15 \pm 0.02 \|^{*}$ & $\left\|^{*} \quad 0.16 \pm 0.02\right\| \dagger$ \\
\hline
\end{tabular}

Values are mean \pm SD. Inspired $\mathrm{O}_{2}$ fraction was 0.6 .

$\mathrm{HR}=$ heart rate; $\mathrm{MAP}=$ mean arterial blood pressure; $\mathrm{CVP}=$ central venous pressure; $\mathrm{MPAP}=$ mean pulmonary artery pressure; $\mathrm{PAWP}=$ pulmonary artery wedge pressure; $\mathrm{CI}=$ cardiac index; $\mathrm{SvO}_{2}=$ mixed venous oxygen saturation; $\mathrm{VO}_{2}=$ oxygen consumption; $\mathrm{SVRI}=$ systemic vascular resistance index; $\mathrm{PVRI}=$ pulmonary vascular resistance index; $\mathrm{Pa}=$ arterial partial pressure; $\mathrm{pHa}=$ arterial $\mathrm{pH} ; \mathrm{Pv}=$ mixed venous partial pressure; $\mathrm{pHv}=$ mixed venous $\mathrm{pH} ; \mathrm{Q}_{\mathrm{S}} / \mathrm{Q}_{\mathrm{T}}=$ shunt fraction; $\mathrm{Pv}-\mathrm{a}=$ mixed venous-arterial partial pressure difference.

${ }^{*} P<0.05$ significantly different versus protocol supine; $+P<0.01$ significantly different versus protocol supine; $\ddagger P<0.01$ significantly different versus semirecumbent; $\S P<0.05$ significantly different versus semirecumbent; $\| P<0.01$ significantly different versus baseline supine.

Vr in all postures (all $P<0.05$ by paired $t$-test). By selecting the larger of these two expiratory Pes-values as reference (see Methods), we eliminated the effect of their posture-related variability on our determinations of test breath-induced changes in Pes (8).

During EIO, mean maximal amplitude of cardiac oscillations in Pes (Fig. 1) was similar in all postures $\left(1.4^{*} \pm 0.6 \mathrm{~cm} \mathrm{H}_{2} \mathrm{O}\right)$, indicating similar magnitude of transmitted intracardiac pressure-changes to the esophageal balloon. During EIO-oscillations, mean Pes-increase rate (Fig. 1) was also stable $\left(9.0^{*} \pm 3.2 \mathrm{~cm}\right.$ $\mathrm{H}_{2} \mathrm{O} / \mathrm{s}$ ) in all postures.

Posture changes did not affect hemodynamic variables. In contrast, pronation resulted in (a) higher $\mathrm{PaO}_{2}$ and lower $\mathrm{Q}_{\mathrm{S}} / \mathrm{Q}_{\mathrm{T}}$ versus all other postures $(P<0.05-$ 0.01 ) and (b) lower $\mathrm{PaCO}_{2}$ and mixed venous $\mathrm{PCO}_{2}$ $\left(\mathrm{PvCO}_{2}\right)$ versus supine $\mathrm{BAS}_{\mathrm{BAS}}$ and supine $\mathrm{PROT}$ (all $\mathrm{P}<$ 0.01) (Table 3; Figs. 3C and D).

During study period, there were no appreciable changes in energy expenditure or metabolic rate determinants such as physiologic stress level (clinical stability was maintained), patient-temperature (37.3* \pm 0.4 , maximal fluctuation always $\leq 0.6^{\circ} \mathrm{C}$ ), feeding, and medication. Also, formula-derived $\mathrm{V}_{2}$ was stable and calculated $\mathrm{R}$ constant; thus, $\mathrm{CO}_{2}$ production should also be stable. During study period, $\mathrm{CO}$ was stable, indicating unchanged $\mathrm{CO}_{2}$-delivery rate to the Ls and time available for alveolar-capillary gas equilibration. Consequently, in each posture, mixed venous-to-arterial $\mathrm{CO}_{2}$ concentration difference $(\mathrm{Cv}$ $\mathrm{aCO}_{2}$ ) reflected $\mathrm{L}-\mathrm{CO}_{2}$ elimination efficiency. Because $\mathrm{CCO}_{2}-\mathrm{PCO}_{2}$ relationship was almost linear at rest and its determinants $\left(\mathrm{pH}, \mathrm{HCO}_{3}\right.$, hemoglobin concentration, and $\mathrm{So}_{2}$ ) (11) were unaffected by posture change, similar $\mathrm{PCO}_{2}$ changes reflected similar $\mathrm{CCO}_{2}$ changes in all postures. Furthermore, in each posture, mixed venous-to-arterial $\mathrm{PCO}_{2}$ difference $\left(\mathrm{Pv}-\mathrm{aCO}_{2}\right)$ reflected $\mathrm{L}-\mathrm{CO}_{2}$ elimination efficiency in that particular posture (12). However, as $\mathrm{L}-\mathrm{CO}_{2}$ excretion also depends on $\mathrm{PvCO}_{2}$ per se (12), we expressed its efficiency as fractional $\mathrm{PvCO}_{2}$ change after the blood passes through the pulmonary circulation $\left(\mathrm{Pv}-\mathrm{aCO}_{2} / \mathrm{PvCO}_{2}\right) \cdot \mathrm{Pv}-\mathrm{aCO}_{2}$ and $\mathrm{PvCO}_{2}$ were higher in prone position versus

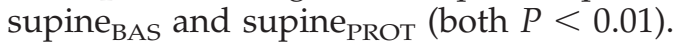

Random posture sequence resulted in five patients being repositioned supine after pronation (Table 4; Fig. 4). Regarding cw mechanics, prone versus supine $_{\text {BAS }}$ and supine ${ }_{\text {POSTPRO }}$ differences were as previously described (Tables 2 and 4; Figs. 3A and B, 4A and $\mathrm{B}$ ); however, Estat, $\mathrm{L}$ was lower only versus supine $_{\text {BAS }}(P<0.01)$. Accordingly, supine ${ }_{\text {POSTPRO versus }}$ supine $_{\text {BAS }}$ resulted in lower Estat,rs and Estat,L $(P<$ 0.05-0.01). Regarding $\mathrm{CO}_{2}$, prone versus supine ${ }_{\mathrm{BAS}}$ and supine ${ }_{\mathrm{POSTPRO}}$ differences were as previously described (Tables 3 and 4; Figs. $3 \mathrm{C}$ and D, 4C and D). However, $Q_{S} / Q_{T}$ was similar in the prone position and supine POSTPRO. Accordingly, supine $_{\text {POSTPRO }}$ versus supine BAS $_{\text {B }}$ resulted in lower $Q_{S} / Q_{T}$ and higher $\mathrm{PaO}_{2}(P<0.05-0.01)$. By contrast, in the five patients in whom supine PROT $_{\text {preceded pronation }}$ (supine $_{\text {PREPRO), all supine }}$ BAS and supine PREPRO versus prone differences in rs mechanics and gas exchange were as above for the whole patient group (data not shown). 
Table 4. Baseline Supine, Postprone Supine, and Prone Respiratory Mechanics During Baseline Ventilation Test Breaths (see also Methods and Table 2) and Gas Exchange Data in Five Study Participants. Gas Exchange Data Correspond to Baseline Ventilation Conditions (see also Methods, Table 2, and Figs. 4C and D)

\begin{tabular}{|c|c|c|c|c|c|c|c|}
\hline \multicolumn{8}{|c|}{ Respiratory mechanics } \\
\hline $\begin{array}{l}\text { Position, } \\
\text { rs component }\end{array}$ & $\begin{array}{c}\mathrm{Rmax}_{\left(\mathrm{cm} \mathrm{H} \mathrm{H}_{2} \mathrm{O} / \mathrm{L} / \mathrm{s}\right)}\end{array}$ & $\begin{array}{l}\mathrm{Rmin} \\
\mathrm{n} \mathrm{H} \mathrm{H}_{2} \mathrm{O} / \mathrm{L}\end{array}$ & $\begin{array}{c}\Delta \mathrm{R} \\
\left(\mathrm{cmH}_{2} \mathrm{O} / \mathrm{L} / \mathrm{s}\right)\end{array}$ & $\begin{array}{c}\text { Edyn } \\
\left(\mathrm{cm} \mathrm{H}_{2} \mathrm{O} / \mathrm{L}\right)\end{array}$ & $\begin{array}{c}\text { Estat } \\
\left(\mathrm{cm} \mathrm{H} \mathrm{H}_{2} \mathrm{O} / \mathrm{L}\right)\end{array}$ & $\begin{array}{c}\Delta \mathrm{E} \\
\left(\mathrm{cm} \mathrm{H} \mathrm{H}_{2} \mathrm{O} / \mathrm{L}\right)\end{array}$ & $\begin{array}{c}\text { PEEPi } \\
\left(\mathrm{cm} \mathrm{H}_{2} \mathrm{O}\right)\end{array}$ \\
\hline Basline supine, rs & $16.6 \pm 2.2$ & $10.9 \pm 2.6$ & $5.6 \pm 0.9$ & $24.9 \pm 2.1$ & $16.4 \pm 1.6$ & $8.6 \pm 1.3$ & $11.9 \pm 1.9$ \\
\hline Baseline supine, $\mathrm{cw}$ & $1.8 \pm 0.7$ & $1.0 \pm 0.3$ & $0.8 \pm 0.5$ & $6.8 \pm 0.8$ & $5.6 \pm 0.9$ & $1.2 \pm 0.8$ & $2.5 \pm 0.6$ \\
\hline Baseline supine, L & $14.8 \pm 1.8$ & $9.9 \pm 2.5$ & $4.9 \pm 1.3$ & $18.1 \pm 2.7$ & $10.8 \pm 1.9$ & $7.4 \pm 2.0$ & $9.4 \pm 1.4$ \\
\hline Postprone supine, rs & $16.0 \pm 2.0$ & $11.0 \pm 2.4$ & $5.0 \pm 0.9$ & $20.1 \pm 2.5$ & $12.6 \pm 1.8 \S$ & $7.5 \pm 1.4$ & $12.6 \pm 2.1$ \\
\hline Postprone supine, cw & $1.8 \pm 0.5$ & $1.0 \pm 0.2$ & $0.8 \pm 0.4$ & $6.8 \pm 0.5$ & $5.6 \pm 0.9$ & $1.2 \pm 0.7$ & $2.5 \pm 0.7$ \\
\hline Postprone supine, L & $14.1 \pm 1.5$ & $9.9 \pm 2.3$ & $4.2 \pm 1.2$ & $13.3 \pm 2.5$ & $7.0 \pm 1.2 \S$ & $6.3 \pm 1.9$ & $10.1 \pm 1.5$ \\
\hline Prone, rs & $20.0 \pm 1.0$ & $12.7 \pm 1.5$ & $7.2 \pm 0.5 \ddagger^{*}$ & $24.4 \pm 0.9$ & $13.4 \pm 0.8$ & $11.0 \pm 0.8+\ddagger$ & $13.1 \pm 2.0$ \\
\hline Prone, $\mathrm{cw}$ & $3.2 \pm 1.0$ & $1.3 \pm 0.6$ & $1.9 \pm 0.6 \ddagger^{*}$ & $10.9 \pm 0.5+\S$ & $8.0 \pm 0.6+\S$ & $2.8 \pm 0.7 \ddagger^{*}$ & $3.4 \pm 0.6$ \\
\hline Prone, L & $16.8 \pm 0.1$ & $11.4 \pm 0.9$ & $5.3 \pm 0.8$ & $13.5 \pm 1.0$ & $5.4 \pm 0.5 \S$ & $8.2 \pm 1.3$ & $9.7 \pm 1.4$ \\
\hline
\end{tabular}

Gas exchange data

\begin{tabular}{lcccccc}
\hline \multicolumn{1}{c}{ Position } & \multicolumn{1}{c}{$\begin{array}{c}\mathrm{PaO}_{2} \\
(\mathrm{~mm} \mathrm{Hg})\end{array}$} & $\begin{array}{c}\mathrm{PaCO}_{2} \\
(\mathrm{~mm} \mathrm{Hg})\end{array}$ & $\begin{array}{c}\mathrm{PvO}_{2} \\
(\mathrm{~mm} \mathrm{Hg})\end{array}$ & $\begin{array}{c}\mathrm{PvCO}_{2} \\
(\mathrm{~mm} \mathrm{Hg})\end{array}$ & $\mathrm{Q}_{\mathrm{S}} / \mathrm{Q}_{\mathrm{T}}$ & ${\mathrm{Pv}-\mathrm{acO}_{2} / \mathrm{PvCO}_{2}}$ \\
\hline Baseline supine & $104 \pm 12$ & $50.4 \pm 1.3$ & $49.1 \pm 2.3$ & $56.0 \pm 0.9$ & $0.28 \pm 0.07$ & $0.10 \pm 0.02$ \\
Postprone supine & $130 \pm 5 \S$ & $46.7 \pm 0.6$ & $52.9 \pm 3.8$ & $52.7 \pm 0.7$ & $0.18 \pm 0.03 \ddagger$ & $0.12 \pm 0.02$ \\
Prone & $204 \pm 11+\S$ & $38.3 \pm 0.9+\S$ & $52.2 \pm 3.4$ & $45.8 \pm 0.7+\S$ & $0.14 \pm 0.03 \S$ & $0.17 \pm 0.03 \S^{*}$ \\
\hline
\end{tabular}

Values are mean \pm sD. Results on hemodynamics and $\mathrm{pH}$ are omitted because they were similar to those presented in Table 3.

$\mathrm{rs}=$ respiratory system; $\mathrm{cw}=$ chest wall; $\mathrm{L}=$ lung; $\mathrm{Rmax}=$ maximal (total) resistance; $\mathrm{Rmin}=$ ohmic resistance; $\Delta \mathrm{R}=\mathrm{additional}$ resistance; $\mathrm{Edyn}=\mathrm{dynamic}$ elastance; Estat $=$ static elastance; $\Delta \mathrm{E}=$ additional elastance; $\mathrm{PEEPi}=$ intrinsic positive end-expiratory pressure; Pa $=$ arterial partial pressure; Pv $=$ mixed venous partial pressure; $Q_{\mathrm{S}} / \mathrm{Q}_{\mathrm{T}}=$ shunt fraction; Pv-a = mixed venous-arterial partial pressure difference.

${ }^{*} P<0.05$ significantly different versus postprone supine; $\dagger P<0.01$ significantly different versus postprone supine; $\ddagger P<0.05$ significantly different versus baseline supine; $\S P<0.01$ significantly different versus baseline supine.

\section{Discussion}

Our main findings were: (a) at baseline $\mathrm{VT}_{\mathrm{T}}$, pronation increases Edyn,cw and Estat,cw and improves arterial oxygenation and $\mathrm{Q}_{\mathrm{S}} / \mathrm{Q}_{\mathrm{T}}$ versus supine and semirecumbent, whereas it also decreases Estat,L and augments $\mathrm{CO}_{2}$ excretion versus supine, (b) regarding $\mathrm{L}$-mechanics at sigh $\mathrm{VT}$, pronation also decreases L-tissue stress relaxation tension and L-time constant inequality (Appendix II) versus supine and semirecumbent positions, (c) in abdominal compression absence, posture change (and especially pronation) does not affect hemodynamic status, (d) Wtot,rs and, thus, overall mechanical ventilation conditions (see Results and Appendix II) are unaffected by posture change, and (e) regarding Estat,L, arterial oxygenation, and $Q_{S} / Q_{T}$, pronation-related benefits versus supine BAS $_{\text {B }}$ seem to be maintained in supine POSTPRO for at least $30-65 \mathrm{~min}$, whereas the reverse is true for $\mathrm{CO}_{2}$ elimination.

We showed that semirecumbent positioning merely increases thoracic-tissue viscoelastic resistance versus supine at baseline Vт (Appendix II; Tables 2 and 3; Figs. 2C and D, 3A); nevertheless, its major benefit versus supine is reduction of nosocomial pneumonia risk (13). In contrast, pronation augments ventilationperfusion $(\mathrm{V} / \mathrm{Q})$ matching and $\mathrm{CO}_{2}$ elimination versus supine; probable contributory factors include increased ribcage elastance (2) and elimination of
L-compression by the heart (14), resulting in decreased Estat,L, attenuated L-inflation gradient, and more homogenous regional and increased total effective alveolar ventilation $\left(\mathrm{V}_{\mathrm{ALV}}\right)$ versus supine $(2,14)$. Pronation proved superior versus semirecumbent with respect to $\mathrm{V} / \mathrm{Q}$ matching but not $\mathrm{CO}_{2}$ elimination; this is consistent with the $\mathrm{cw} / \mathrm{L}$ mechanics similarities in semirecumbent and supine positions but suggests a slightly increased effective $\mathrm{V}_{\mathrm{ALV}}$ in the former.

In supine $\mathrm{POSTPRO}_{\mathrm{P}}, \mathrm{CO}_{2}$ elimination decreased toward baseline (Table 4, Figs. 4C and D), indicating accentuated L-inflation gradient versus prone, causing a decrease in effective $\mathrm{V}_{\mathrm{ALV}}$; accordingly, $\mathrm{cW}$ mechanics were similar versus supine $_{\mathrm{BAS}}$; however, arterial oxygenation and $\mathrm{Q}_{\mathrm{S}} / \mathrm{Q}_{\mathrm{T}}$ were still improved versus supine $\mathrm{BAS}_{\text {(Table 4, }}$ Figs. $4 \mathrm{C}$ and $\mathrm{D}$ ), indicating partial maintenance of pronations augmented $V_{A L V}$ homogeneity and $V / Q$ matching; this is consistent with enhanced dorsal L-region recruitment during tidal $\mathrm{L}$-inflation versus supine ${ }_{\mathrm{BAS}}$ because of the preceding pronation effects (2).

Some may argue that our results on pronation were mainly because of posture change-induced mobilization of tracheobronchial secretions. However, this cannot explain the partial reversal of pronation benefits in

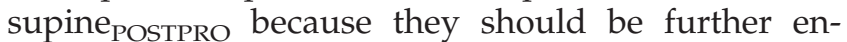
hanced by further secretion mobilization secondary to further posture change. 


\section{Tidal Volume $=0.6 \mathrm{~L}$}

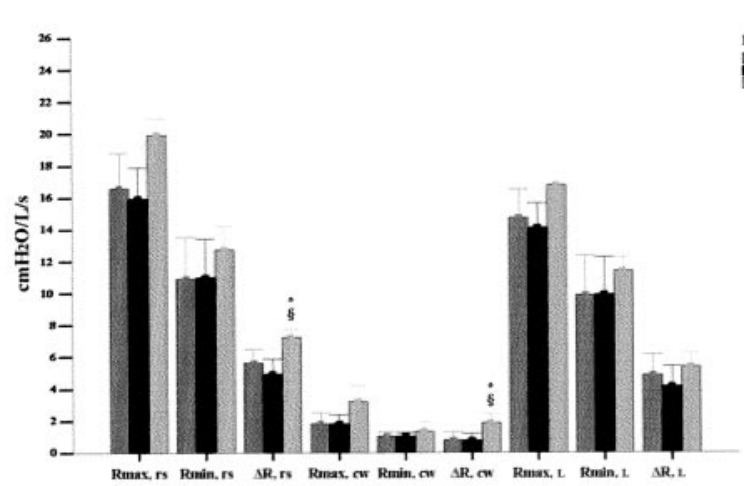

A

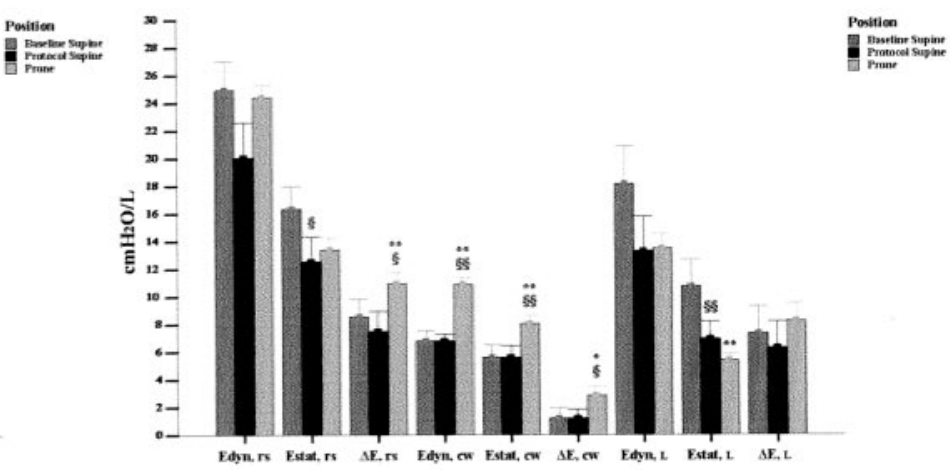

B

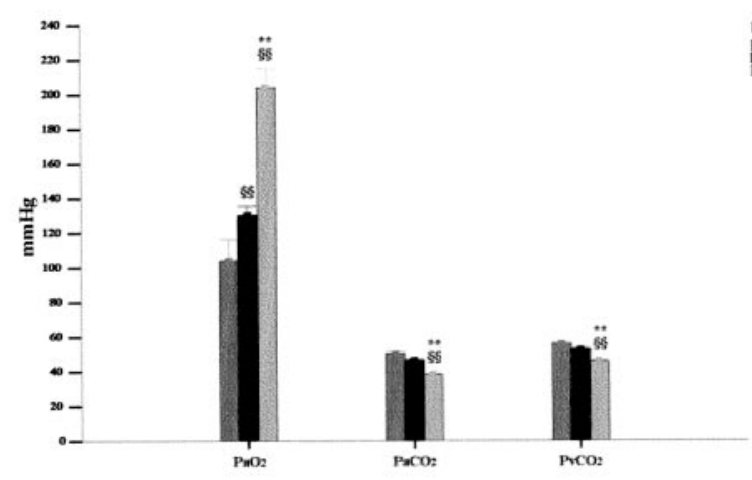

C
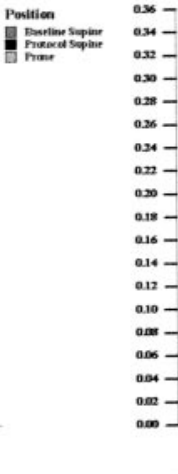

D

Figure 4. Results on resistance, elastance, and gas exchange obtained from the postprone supine subgroup. Bars show mean values, and error bars show mean + SD; precise numerical values are given in Table 4 . The presentation includes only variables that exhibited at least one posture shift-related significant change in the whole study group and the aforementioned subgroup (Tables 2-4; Fig. 3). (A-D) Results on resistance, elastance, blood gases, and $\mathrm{CO}_{2}$ elimination and shunt fraction (respectively) obtained at baseline $(0.6 \mathrm{~L})$ tidal volume. rs $=$ respiratory system; $\mathrm{cw}=$ chest wall; $\mathrm{L}=$ lung; $\mathrm{Rmax}=$ maximal (total) resistance; $\mathrm{Rmin}=$ ohmic resistance; $\Delta \mathrm{R}=$ additional resistance due to tissue stress relaxation tension or L-time constant inequalities; Edyn $=$ dynamic elastance; Estat $=$ static elastance; $\Delta \mathrm{E}=$ additional elastance due to tissue stress relaxation tension or L-time constant inequalities; $\mathrm{Pa}=$ arterial gas partial pressure; $\mathrm{Pv}=$ mixed venous gas partial pressure; $\mathrm{Pv}-\mathrm{a}=$ mixed venous-to-arterial gas partial pressure difference; $\mathrm{Q}_{\mathrm{S}} / \mathrm{Q}_{\mathrm{T}}=$ shunt fraction. In the presented figure, elastance and resistance variables of each rs-component are abbreviated as already defined variable abbreviation, rs component (e.g., maximal [total] resistance $[R \max ]$ of the $\mathrm{rs}, \mathrm{Rmax}, \mathrm{rs}) . \S / \S \S=$ significantly different versus baseline supine, $P<0.05 /<0.01$ (respectively); ${ }^{*} * *=$ significantly different versus protocol supine, $P<0.05 /<0.01$ (respectively).

The pronation-induced reduction in L-time constant inequality during sigh-Vт EIO (Appendix II; Table 2; Figs. 2D, 3E and F) suggests a reduced number of L-units with very low (tending toward 0) or high (tending toward infinity) time constants and, thus, a reduced number of atelectatic and hyperinflated alveoli, respectively; this further suggests increased sigh effectiveness and reduced alveolar-rupture probability. Furthermore, the observed hemodynamic and ventilation-conditions' stability and absence of posture-shift-related complications in conjunction with the rest of our results demonstrate prone position's applicability, effectiveness, and benefits versus semirecumbent positioning.

Posture sequence randomization enabled posturedata determinations and comparisons without risk of a certain posture order systematically influencing results obtained in a subsequent one. Data on a postsemirecumbent supine position were not obtained for comparison with our postprone data; however, available and presented data (Table 2; Fig. 3) strongly suggest lack of semirecumbent-related 
benefit after supine posture resumption. Consequently, our methodology has enabled us to produce results leading to satisfactory conclusions regarding pronation merits.

Pes-measurements reliability could be questionable because after pronation, alveolar pressure transmission to the esophagus may vary as the heart moves ventrally (2). A change in heart positioning relative to the esophagus or esophageal-balloon should also affect transmission of intracardiac pressure changes to the latter. However, myocardial wall motion pattern, contractility, and cyclic intracardiac pressure changes should have remained stable in all postures (see Results; Table 3); consequently, our EIO-cardiac oscillation data suggest unchanged transmission pattern of intracardiac pressure changes to the esophageal balloon. Thus, the initial correct esophageal-balloon positioning relative to the heart was probably maintained throughout the study period, and respiratory cycle-induced Pes-changes were measured as accurately as possible in all postures $(2,15)$.

A severe limitation was that no systematic data

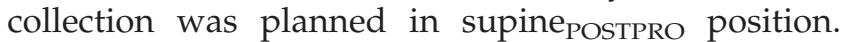
This was counterbalanced by posture random order resulting in available-for-comparison supine ${ }_{\text {POSTPRO }}$ data in five participants. Because all determined variables exhibited similar response-patterns to posture

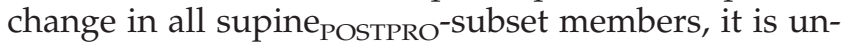
likely that the small size of the latter had significantly affected the results (16); this is also true for the whole study group. However, we cannot totally exclude type II errors (2).

EELV, physiologic and alveolar dead space and energy expenditure were not directly determined. The former two limitations were partially counterbalanced by our determinations of $\triangle$ EELV (increment in functional residual capacity because of expiratory flow limitation) (6) and $\mathrm{Pv}_{-} \mathrm{aCO}_{2} / \mathrm{PvCO}_{2}$ (which reflected effective $\mathrm{V}_{\mathrm{ALV}}$ during hemodynamic and probable metabolic stability) (see Results). Finally, $\mathrm{O}_{2}$ measurements with metabolic monitors may exhibit inaccuracies at $\mathrm{FIO}_{2}>0.5$ (17).

Noninvasive positive-pressure ventilation (NIPPV) is recommended as first-line treatment in ARF-COPD patients (18-21). NIPPV may avert invasive mechanical ventilation (IMV) in 50\%-75\% of cooperative, alert, hemodynamically stable patients $(18-21)$. However, a meta-analysis (22) revealed an overall NIPPV-induced IMV-reduction of only $18 \%$ in an ARF-COPD cohort, originating from 15 randomized-controlled trials (RCTs). Also, approximately $80 \%$ of ARF-COPD patients admitted to a university-affiliated intensive care unit required IMV (23). ARF-COPD patients exhibit an in-hospital mortality of $24 \%$ (24), which may be reduced by using lower VTs during IMV (and thus, decreasing the incidence of hemodynamic compromise and ventilatorinduced lung injury [VILI]) (23). Interestingly, recent evidence suggests that intermittent pronation may also reduce VILI-risk (25). Our findings of pronation-induced improvement in L-mechanical behavior (Table 2; Figs. $3 \mathrm{~A}, \mathrm{~B}, \mathrm{E}, \mathrm{F})$ are consistent with reduced VILI risk; furthermore, we demonstrated a pronation-induced improvement in gas exchange efficiency (i.e., L-function) (Tables 2 and 3; Figs. 3C and D), partially maintained along with pronation Estat,L-reduction (improvement in

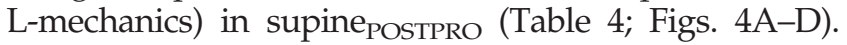
Thus, pronation may constitute a useful therapeutic strategy in the management of ARF-COPD patients, and RCTs comparing outcomes of COPD patients treated with prone and semirecumbent positioning are warranted.

\section{Appendix I}

\section{Formulas used to derive hemodynamic and gas exchange variables ${ }^{1}$}

1. Cardiac index $=\mathrm{CO} / \mathrm{BSA}$.

2. Systemic vascular resistance index $=(\mathrm{MAP}-\mathrm{CVP})$. $80 / \mathrm{CI}$.

3. Pulmonary vascular resistance index $=(\mathrm{MPAP}-$ PAWP) $\cdot 80 / C I$.

4. $\mathrm{O}_{2}$ consumption per $\mathrm{m}^{2} \mathrm{BSA}=\mathrm{CI} \cdot 1.36 \cdot \mathrm{Hgb}$. $\left(\mathrm{SaO}_{2}-\mathrm{SvO}_{2}\right)$.

5. Respiratory quotient $=($ FEY of carbohydrate intake) $\cdot 1.0+($ FEY of protein intake $) \cdot 0.8+($ FEY of lipid intake) $\cdot 0.7 .^{2}$

6. Alveolar $\mathrm{PO}_{2}=\mathrm{PIO}_{2}-\mathrm{P}_{\mathrm{A}} \mathrm{CO}_{2} \cdot\left(\mathrm{FIO}_{2}-\left(1-\mathrm{FIO}_{2}\right)\right.$. $\left.\mathrm{R}^{-1}\right) ; \mathrm{PIO}_{2}=\mathrm{FIO}_{2} \cdot\left(\mathrm{P}_{\mathrm{B}}-47\right) ; \mathrm{P}_{\mathrm{A}} \mathrm{CO}_{2} \sim \mathrm{PaCO}_{2}$.

7. $\mathrm{O}_{2}$ content of blood $=\mathrm{Hgb} \cdot 1.36 \cdot \mathrm{SO}_{2} / 10+$ $0.003 \cdot \mathrm{PO}_{2}$.

8. Shunt fraction $=\left(\mathrm{CCO}_{2}-\mathrm{CaO}_{2}\right) /\left(\mathrm{CCO}_{2}-\mathrm{CvO}_{2}\right)$.

$\mathrm{CO}=$ cardiac output $(\mathrm{L} / \mathrm{min}) ; \mathrm{BSA}=$ body surface area $\left(\mathrm{m}^{2}\right) ; \mathrm{MAP}=$ mean arterial blood pressure $(\mathrm{mm}$ $\mathrm{Hg}) ; \mathrm{CVP}=$ central venous pressure $(\mathrm{mm} \mathrm{Hg}) ; \mathrm{CI}=$ cardiac index $\left(\mathrm{L} \cdot \mathrm{min}^{-1} \cdot \mathrm{m}^{-2}\right) ; 80=$ transformation factor of Wood units $\left(\mathrm{mm} \mathrm{Hg} \cdot \mathrm{L}^{-1} \cdot \mathrm{min}\right)$ to standard metric units (dynes $\cdot \mathrm{s} \cdot \mathrm{cm}^{-5}$ ); MPAP $=$ mean pulmonary artery blood pressure ( $\mathrm{mm} \mathrm{Hg}$ ); PAWP, pulmonary artery wedge pressure $(\mathrm{mm} \mathrm{Hg}) ; \mathrm{Hgb}=$ hemoglobin concentration in $\mathrm{g} / \mathrm{L} ; 1.36=\mathrm{O}_{2}$ combining power of $1 \mathrm{~g}$ of hemoglobin $(\mathrm{mL}) ; \mathrm{SaO}_{2}=$ arterial $\mathrm{O}_{2}$ saturation; $\mathrm{SvO}_{2}=$ mixed venous $\mathrm{O}_{2}$ saturation; $\mathrm{FEY}=$ fractional energy yield relative to total of pre-scribed nutritional support; $\mathrm{P}=$ gas partial pressure $(\mathrm{mm} \mathrm{Hg})$; $\mathrm{PIO}_{2}=$ inspired $\mathrm{O}_{2}$ partial pressure $(\mathrm{mm} \mathrm{Hg}) ; \mathrm{P}_{\mathrm{A}} \mathrm{CO}_{2}$ $=$ alveolar $\mathrm{CO}_{2}$ partial pressure $(\mathrm{mm} \mathrm{Hg}) ; \mathrm{FIO}_{2}=$ inspired $\mathrm{O}_{2}$ fraction; $\mathrm{R}=$ respiratory quotient; $\mathrm{P}_{\mathrm{B}}=$

\footnotetext{
${ }^{1}$ Sources: Mark JB, Slaughter TF, Reves JG. Cardiovascular monitoring. In: Miller RD, ed. Anesthesia. 5th ed. New York: Churchill Livingstone, 2000:1117-230, and Moon ME, Camporesi EM. Respiratory monitoring. In: Miller RD, ed. Anesthesia. 5th ed. New York: Churchill Livingstone, 2000:1255-96.

${ }^{2}$ Source: Reference 17.
} 
barometric pressure $(\mathrm{mm} \mathrm{Hg}) ; 47$ = water saturated vapor pressure at $37^{\circ} \mathrm{C}(\mathrm{mm} \mathrm{Hg}) ; 0.003=\mathrm{O}_{2}$ solubility coefficient at $37^{\circ} \mathrm{C}\left(\mathrm{mL} \cdot \mathrm{dL}^{-1} \cdot \mathrm{mm} \mathrm{Hg}\right) ; \mathrm{PO}_{2}=\mathrm{O}_{2}$ partial pressure $(\mathrm{mm} \mathrm{Hg}) ; \mathrm{CcO}_{2} / \mathrm{CaO}_{2} / \mathrm{CvO}_{2}=\mathrm{O}_{2}$ content in end-capillary/arterial/mixed-venous blood (respectively).

\section{Appendix II}

\section{Relationships among inspiratory work subcomponents and elastance, resistance, and intrinsic positive end-expiratory pressure (PEEPi). ${ }^{3}$}

1. Total inspiratory work reflects in combination PEEPi during baseline mechanical ventilation (see Methods) and ohmic resistance (Rmin), additional resistance $(\Delta R),{ }^{4}$ caused by time constant inequality within the lung or tissue stress relaxation tension and static elastance (Estat) as tidal volume varies from 0.2 to $1.2 \mathrm{~L}$ (by $0.2 \mathrm{~L}$ increments) with square-wave inspiratory flow kept constant at $0.91 \mathrm{~L} / \mathrm{s}$ (protocol test breathing (PTB) described in Methods). PTB.

2. Resistive inspiratory work reflects Rmin during

3. Additional dynamic work reflects $\Delta \mathrm{R}$ and $\Delta \mathrm{E}$ during PTB.

4. Elastic inspiratory work reflects Estat during PTB.

5. PEEPi inspiratory work reflects PEEPi during baseline mechanical ventilation.

\section{References}

1. Nakos G, Tsangaris I, Kostanti E, et al. Effect of the prone position on patients with hydrostatic pulmonary edema compared with patients with acute respiratory distress syndrome and pulmonary fibrosis. Am J Respir Crit Care Med 2000;161: 360-8.

2. Pelosi $\mathrm{P}$, Tubiolo D, Mascheroni D, et al. Effects of the prone position on respiratory mechanics and gas exchange during acute lung injury. Am J Respir Crit Care Med 1998;157:387-93.

3. Patroniti N, Foti G, Cortinovis B, et al. Sigh improves gas exchange and lung volume in patients with acute respiratory distress syndrome undergoing pressure support ventilation. Anesthesiology 2002;96:788-94.

4. American Thoracic Society. Standards for the diagnosis and care of patients with chronic obstructive pulmonary disease. Am J Respir Crit Care Med 1995;152:S77-121.

5. Dewan NA, Rafique S, Kanwar B, et al. Acute exacerbation of COPD: factors associated with poor treatment outcome. Chest 2000;117:662-71.

\footnotetext{
${ }^{3}$ Sources: Reference 6 and Eissa NT, Ranieri VM, Correil C, et al. Analysis of behavior of the respiratory system in ARDS patients: effects of flow, volume, and time. J Appl Physiol 1991;70:2719-29.

${ }^{4}$ Related to additional elastance $(\Delta \mathrm{E})$ according to the following formula: $\Delta \mathrm{E}=\Delta \mathrm{R} /$ inspiratory time.
}

6. Coussa ML, Guerin C, Eisa T, et al. Partitioning of work of breathing in mechanically ventilated COPD patients. J Appl Physiol 1993;75:1711-9.

7. Baydur AP, Behrakis PK, Zin WA, et al. A simple method for assessing the validity of the esophageal balloon technique. Am Rev Respir Dis 1982;126:788-91.

8. Polese G, Rossi A, Appendini L, et al. Partitioning of respiratory mechanics in mechanically ventilated patients. J Appl Physiol 1991;71:2425-33.

9. Lapinsky SE, Aubin M, Mehta S, et al. Safety and efficacy of sustained inflation for alveolar recruitment in adults with respiratory failure. Intensive Care Med 1999;25:1297-301.

10. Pappert D, Rossaint $K$, Slama K, et al. Influence of positioning on ventilation-perfusion relationships in severe adult respiratory distress syndrome. Chest 1994;106:1511-6.

11. Sun $X G$, Nansen JE, Stringer WW, et al. Carbon dioxide pressure-concentration relationship in arterial and mixed venous blood during exercise. J Appl Physiol 2001;90:1798-810.

12. Wiklund L, Jorfeldt L, Stjernstrom H, Rubertsson S. Gas exchange as monitored in mixed venous and arterial blood during experimental cardiopulmonary resuscitation. Acta Anaesthesiol Scand 1992;36:427-35.

13. Drukalovic MB, Torres A, Bauer TT, et al. Supine body position as a risk factor for nosocomial pneumonia in mechanically ventilated patients: a randomized trial. Lancet 1999;354:1851-8.

14. Albert RK, Hubmayr RD. The prone position eliminates compression of the lungs by the heart. Am J Respir Crit Care Med 2000;161:1660-5.

15. Pelosi $\mathrm{P}$, Croci $\mathrm{M}$, Calappi E, et al. The prone positioning during general anesthesia minimally affects respiratory mechanics while improving functional residual capacity and increasing oxygen tension. Anesth Analg 1995;80:955-60.

16. Pelosi P, Croci M, Calappi E, et al. Prone positioning improves pulmonary function in obese patients during general anesthesia. Anesth Analg 1996;83:578-83.

17. Marino PL. Nutrient and energy requirements. In: Marino PL, ed. The ICU book. 2nd ed. Baltimore, MD: Williams \& Wilkins, 1997:721-36.

18. Mehta S, Hill NS. Noninvasive ventilation. Am J Respir Crit Care Med 2001;163:540-77.

19. American Thoracic Society. International Consensus Conferences in Intensive Care Medicine: noninvasive positive pressure ventilation in acute respiratory failure. Am J Respir Crit Care Med 2001;163:283-91.

20. Brochard L, Mancebo J, Wysocki M, et al. Noninvasive ventilation for acute exacerbations of chronic obstructive pulmonary disease. N Engl J Med 1995;333:817-22.

21. Hilbert G, Vargas F, Valentino R, et al. Noninvasive ventilation in acute exacerbations of chronic obstructive pulmonary disease in patients with and without home noninvasive ventilation. Crit Care Med 2002;30:1453-8.

22. Peter JV, Moran JL, Phillips-Hughes J, Warn D. Noninvasive ventilation in acute respiratory failure: a meta-analysis update. Crit Care Med 2002;30:555-62.

23. Afessa B, Morales IJ, Scanlon PD, Peters SG. Prognostic factors, clinical course, and hospital outcome of patients with chronic obstructive pulmonary disease admitted to an intensive care unit for acute respiratory failure. Crit Care Med 2002;30:1610-5.

24. Seneff MG, Wagner DP, Wagner RP, et al. Hospital and 1-year survival of patients admitted to intensive care units with acute exacerbation of chronic obstructive pulmonary disease. JAMA 1995;20:1852-7.

25. Beuret $\mathrm{P}$, Carbon MJ, Nounrdine $\mathrm{K}$, et al. Prone position as prevention of lung injury in comatose patients: a prospective, randomized controlled study. Intensive Care Med 2002;28: $564-9$. 